Homology, Homotopy and Applications, vol.15(2), 2013, pp.103-125

\title{
THE WHITEHEAD TYPE THEOREMS IN COARSE SHAPE THEORY
}

\author{
NIKOLA KOCEIĆ BILAN AND NIKICA UGLEŠIĆ
}

(communicated by Alexander Mishchenko)

\begin{abstract}
The analogues of Whitehead's theorem in coarse shape theory, i.e., in the pointed coarse pro-category $\mathrm{pro}^{*}-\mathrm{HPol}_{0}$ and in the pointed coarse shape category $S h_{0}^{*}$, are proved. In other words, if a pointed coarse shape morphism of finite shape dimensional spaces induces isomorphisms (epimorphism, in the top dimension) of the corresponding coarse $k$-dimensional homotopy pro-groups, then it is a pointed coarse shape isomorphism.
\end{abstract}

\section{Introduction}

The classical Whitehead theorem (Theorem 1 of $[\mathbf{2 7}]$ ) is one of the few most important theorems in algebraic topology, especially homotopy theory. It is not (except in some special cases) a kind of a theorem that allows one to do something in an easier or more efficient way. Its significance is much deeper since it relates rather different mathematical branches: geometry (topology, especially homotopy theory) to algebra (group theory). Namely, it proves that the main goal of the homotopy theory, i.e., the homotopy type classification of locally nice spaces, via the homotopy equivalences, can be achieved by purely algebraic tools. However, the range of application of the Whitehead theorem is strictly limited to the class of all connected (pointed) spaces having the homotopy types of $C W$-complexes. Thus, when the notion of shape was introduced, i.e., when the homotopy theory was suitably generalized to the shape theory (for all topological spaces), the great importance of an appropriate generalization of the Whitehead theorem became obvious by itself.

The first step in the generalization of the Whitehead theorem to the shape theory was made by M. Moszyńska [22], by proving an appropriate analogue for finitedimensional metric compacta. After that, she exhibited an analogous theorem for movable pointed spaces [23]. S. Mardešić proved the Whitehead theorem for topological spaces when a shape morphism is induced by a (continuous) map [15], as well as a homological and cohomological version of the "Whitehead theorem" in shape theory [16]. A slightly better result was obtained by K. Morita [20]. The full result was first proved also by K. Morita [21]. Some cases with more general or special con-

Received December 28, 2012, revised March 10, 2013, May 28, 2013; published on November 16, 2013.

2000 Mathematics Subject Classification: 55P55, 55Q05, 55N99.

Key words and phrases: inverse system, pro-category, pro*-category, expansion, shape, coarse shape, homotopy pro-group, $m$-equivalence.

Article available at http://intlpress.com/HHA/v15/n2/a6 and doi:10.4310/HHA.2013.v15.n2.a6

Copyright (C) 2013, International Press. Permission to copy for private use granted. 
ditions were considered by several other authors: J. Dydak $[\mathbf{1}, \mathbf{2}, \mathbf{3}]$, J. Keesling $[\mathbf{6}]$, D. A. Edwards and R. Geoghegan $[\mathbf{4}, \mathbf{5}]$, and some others. For instance, J. Dydak generalized the Whitehead theorem by replacing dimension by shape dimension. All the main results can be found in the book of S. Mardešić and J. Segal [18, Chapter II, Section 5].

The coarse shape theory was founded by the authors in $[\mathbf{1 2}]$. Its roots reach $[\mathbf{1 7}]$ and $[\mathbf{7}]$, where the problem of shape types of fibers of a shape fibration of metric continua was considered and solved: They need not to be of the same shape type; nevertheless, they all are of the same $S$-type [17] (and $S^{*}$-type [19]). In [19] S. Mardešić and the second named author succeeded in characterizing the $S^{*}$-type in a category framework. So it was the starting point of the coarse shape theory for compact metrizable spaces. However, a suitable generalization to all topological spaces required an essential redefinition (characterization) of morphisms [12]. The coarse shape theory enables a classification of topological spaces strictly coarser than the shape type classification. Nevertheless, almost all important shape invariants are, actually, the invariants of coarse shape. The category framework of the coarse shape provides some new topological invariants (the coarse shape path connectedness [13]) and algebraic invariants (the coarse shape groups [11]).

In this paper, after naturally defining the notions of an $m$-equivalence of pro*- $^{*}$ HTop $_{0}$ and a coarse (shape) m-equivalence, we have generalized the Whitehead theorem to the coarse shape theory for topological spaces. In the realizing category pro $^{*}-\mathrm{HPol}_{0}$ of the coarse shape category for pointed topological spaces (i.e., in the standard pointed coarse pro-category), it reads as follows (see Theorem 4.1):

Let $m \in \mathbb{N}$, and let $\left(\boldsymbol{X}, \boldsymbol{x}_{0}\right)$ and $\left(\boldsymbol{Y}, \boldsymbol{y}_{0}\right)$ be inverse systems of pointed connected polyhedra (in $\mathrm{HPol}_{0}$ ) such that, for every $\lambda \in \Lambda$, $\operatorname{dim} X_{\lambda} \leqslant m-1$, and, for every $\mu \in M, \operatorname{dim} Y_{\mu} \leqslant m$. If $f^{*}:\left(\boldsymbol{X}, \boldsymbol{x}_{0}\right) \rightarrow\left(\boldsymbol{Y}, \boldsymbol{y}_{0}\right)$ is an m-equivalence of pro $^{*}-\mathrm{HPol}_{0}$, then $f^{*}$ is an isomorphism.

In the (pointed) coarse shape category $S h_{0}^{*}$, the statement is as follows (see Theorem 4.3):

Let $m \in \mathbb{N}$, and let $\left(X, x_{0}\right)$ and $\left(Y, y_{0}\right)$ be connected pointed spaces satisfying sd $X \leqslant m-1$ and sd $Y \leqslant m$. If $F^{*}:\left(X, x_{0}\right) \rightarrow\left(Y, y_{0}\right)$ is a coarse m-equivalence of $S h_{0}^{*}$, then $F^{*}$ is an isomorphism.

As a conclusion, roughly speaking, this paper shows that the classification of finite shape dimensional spaces via the appropriate coarse shape equivalences is an algebraic problem.

We also remind that the full analogue of the Hurewicz isomorphism theorem in the coarse shape theory is already proved by the first named author ([10, Theorem 4.11]; compare the full shape analogue in [18, Theorem II.4.1.1]). We believe that the results and techniques of this paper could help in the further studying of some important (algebraic) properties of spaces and mappings. However, since the technique is very sophisticated the progress is rather slow. For instance, one is still searching for an appropriate definition of the "coarse shape fibration" of topological spaces. 


\section{Preliminaries}

\subsection{The coarse shape category and functor}

By following [12], for every category pair $(\mathcal{C}, \mathcal{D})$, where $\mathcal{D} \subseteq \mathcal{C}$ is a pro-reflective subcategory, i.e., every $\mathcal{C}$-object $X$ admits a $\mathcal{D}$-expansion $\boldsymbol{p}: X \rightarrow \boldsymbol{X}$ ([18, I.2.1-3]; since [25], the term "dense" has been replaced by "pro-reflective"!), there exists an abstract coarse shape category $S h_{(\mathcal{C D})}^{*}$, which is constructed in the same general way as the shape category $S h_{(\mathcal{C}, \mathcal{D})}$. The difference is in the replacement of the ordinary pro-category pro-D (the realizing category for $S h_{(\mathcal{C}, \mathcal{D})}$ ) by the new "*-pro-category" pro*- $^{*} \mathcal{D}$, termed the coarse pro-category.. The object class

$$
O b\left(S h_{(\mathcal{C D})}^{*}\right)=O b(\mathcal{C})
$$

while

$$
\operatorname{Sh}_{(\mathcal{C D})}^{*}(X, Y) \approx p r o^{*}-\mathcal{D}(\boldsymbol{X}, \boldsymbol{Y})
$$

( $\boldsymbol{X}$ and $\boldsymbol{Y}$ are $\mathcal{D}$-expansions). In other words, a coarse shape morphism of $X$ to $Y$, denoted by $F^{*}: X \rightarrow Y$, is the equivalence class $\left\langle\boldsymbol{f}^{*}\right\rangle$ of an appropriate morphism $f^{*}: \boldsymbol{X} \rightarrow \boldsymbol{Y}$ of pro $^{*}-\mathcal{D}$.

A brief description of the coarse pro-category pro* $^{*}-\mathcal{A}$ ", for an arbitrary category $\mathcal{A}$, and the corresponding functor $\underline{J}:$ pro- $\mathcal{A} \rightarrow$ pro $^{*}-\mathcal{A}$ reads as follows. In the first step we construct the category $i n v^{*}-\mathcal{A}$ by an essential enlarging of the morphism class of $i n v-\mathcal{A}$. Given a pair of inverse systems in $\mathcal{A}, \boldsymbol{X}=\left(X_{\lambda}, p_{\lambda \lambda^{\prime}}, \Lambda\right), \boldsymbol{Y}=\left(Y_{\mu}, q_{\mu \mu^{\prime}}, M\right)$, a *-morphism (originally an $S^{*}$-morphism $[\mathbf{1 2}]$ ) of inverse systems, $\left(f, f_{\mu}^{n}\right): \boldsymbol{X} \rightarrow \boldsymbol{Y}$, consists of a function $f: M \rightarrow \Lambda$ (the index function) and, for each $\mu \in M$, of a sequence of $\mathcal{A}$-morphisms $f_{\mu}^{n}: X_{f(\mu)} \rightarrow Y_{\mu}, n \in \mathbb{N}$, satisfying a certain commutativity condition. The composition of the $*$-morphisms and the identities are defined in the most natural way. In the second step we define an appropriate equivalence relation (on each morphism set $\left.i n v^{*}-\mathcal{A}(\boldsymbol{X}, \boldsymbol{Y})\right),\left(f, f_{\mu}^{n}\right) \sim\left(f^{\prime}, f_{\mu}^{\prime n}\right)$, which is compatible with the category composition. Therefore, there exists the corresponding quotient category

$$
\operatorname{pro}^{*}-\mathcal{A}=\left(i n v^{*}-\mathcal{A}\right) /(\sim) .
$$

Further, for every category $\mathcal{A}$, there exists a (faithful) functor

$$
\underline{J}: \text { pro- } \mathcal{A} \rightarrow \text { pro }^{*}-\mathcal{A}
$$

defined by $\underline{J}(\boldsymbol{X})=\boldsymbol{X}$ and, for an $\boldsymbol{f}=\left[\left(f, f_{\mu}\right)\right], \underline{J}(\boldsymbol{f})=\boldsymbol{f}^{*}=\left[\left(f, f_{\mu}^{n}\right)\right]$, where, for every $n \in \mathbb{N}, f_{\mu}^{n}=f_{\mu}$, for all $\mu \in M$. (Such an $\left(f, f_{\mu}^{n}=f_{\mu}\right)$ is called an induced *-morphism.) Especially, for a pro-reflective subcategory $\mathcal{D} \subseteq \mathcal{C}$, there exists a faithful functor $\underline{J}:$ pro- $\mathcal{D} \rightarrow$ pro $^{*}-\mathcal{D}$ described above. It induces a faithful functor

$$
J: S h_{(\mathcal{C}, \mathcal{D})} \rightarrow S h_{(\mathcal{C}, \mathcal{D})}^{*}
$$

(relating the shape and the coarse shape category), which keeps the objects fixed.

The most interesting (standard) example of the coarse shape category $S h_{(\mathcal{C}, \mathcal{D})}^{*} \equiv$ $S h^{*}$ is given by $\mathcal{C}=$ HTop (the homotopy category of topological spaces) and $\mathcal{D}=$ HPol (the homotopy category of polyhedra) or $\mathcal{D}=H A N R$ (the homotopy category of ANR's for metric spaces). In the special (sub)case of $\mathcal{C}=H c M$ (the homotopy category of compact metrizable spaces) and $\mathcal{D}=\mathrm{HcPol}$ (the homotopy category of compact polyhedra) or $\mathcal{D}=H c A N R$ (the homotopy category of compact ANR's), only the sequential expansions are required. 
Besides the standard (absolute) case $S h^{*}$, we shall also need the standard pointed case $S h_{0}^{*}\left(\mathcal{C}=\mathrm{HTop}_{0}, \mathcal{D}=\mathrm{HPol}_{0}\right.$ or $\left.H A N R_{0}\right)$.

\subsection{The induced $p r o^{*}$-homotopy group functors}

Let $\mathcal{C}=\mathrm{HTop}_{0}, \mathcal{D}=\mathrm{HPol}_{0}$, and $\mathcal{K}=$ Set $_{0}$ (the category of pointed sets) or $\mathcal{K}=$ $\operatorname{Grp}$ (the category of groups). Then, by following Section 3 of [10], the $k$-th homotopy group functor $U \equiv \pi_{k}: \mathrm{HPol}_{0} \rightarrow G r p, k \in\{0\} \cup \mathbb{N},\left(U \equiv \pi_{0}: \mathrm{HPol}_{0} \rightarrow S_{\text {Set }}\right)$, which assigns the $k$-th homotopy group $\pi_{k}\left(P, p_{0}\right)$ (pointed set $\left.\pi_{0}\left(P, p_{0}\right)\right)$ to every pointed polyhedron $\left(P, p_{0}\right)$ and the homomorphism $f_{k}=\pi_{k}(f):\left(P, p_{0}\right) \rightarrow\left(Q, q_{0}\right)$ to each $H$-map $[f]:\left(P, p_{0}\right) \rightarrow\left(Q, q_{0}\right)$, induces the functor

$$
\text { pro-U } \equiv \text { pro- } \pi_{k}: S h_{0} \rightarrow \text { pro-Grp }\left(\text { pro-U } \equiv \text { pro- }_{0}: S h_{0} \rightarrow \text { pro-Set }_{0}\right),
$$

called the induced $k$-th pro-homotopy group functor on the pointed shape category, as well as

$$
\begin{aligned}
& \text { pro }^{*}-U \equiv \text { pro }^{*}-\pi_{k}: S h_{0}^{*} \rightarrow \text { pro }^{*}-G r p \\
& \left(\text { pro* }^{*}-U \equiv \text { pro }^{*}-\pi_{0}: S h_{0}^{*} \rightarrow \text { pro }^{*}-S e t_{0}\right)
\end{aligned}
$$

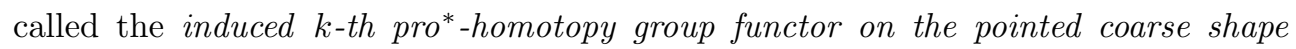
category into the coarse pro-category of groups (pointed sets, for $k=0$ ). The functor pro $^{*}-\pi_{k}$ is an extensions of pro- $\pi_{k}, k \in\{0\} \cup \mathbb{N}$, in the sense that $\left(\right.$ pro $\left.^{*}-\pi_{k}\right) J=$ $\underline{J}\left(\right.$ pro- $\left.\pi_{k}\right)$ holds, where $J$ and $\underline{J}$ are the corresponding embedding functors. An application is demonstrated by Example 3.6 of [10].

\section{3. m-equivalence of pro* $^{*}-\mathrm{HTop}_{0}$}

We first define a coarse pro-analogue of an $n$-equivalence as follows (compare $[\mathbf{2 4}$, p. 404] and the introducing note above Theorem II.5.2.1 of [18]).

Definition 3.1. Let $m \in \mathbb{N}$. A morphism $\boldsymbol{f}^{*}:\left(\boldsymbol{X}, \boldsymbol{x}_{0}\right) \rightarrow\left(\boldsymbol{Y}, \boldsymbol{y}_{0}\right)$ of pro $^{*}$-HTopo ${ }_{o}$ is said to be an $m$-equivalence of pro $^{*}-\mathrm{HTop}_{0}$ if the induced morphism

$$
\boldsymbol{\pi}_{k}^{*}\left(\boldsymbol{f}^{*}\right) \equiv \boldsymbol{f}_{k}^{*}: \boldsymbol{\pi}_{k}^{*}\left(\boldsymbol{X}, \boldsymbol{x}_{0}\right)=\boldsymbol{\pi}_{k}\left(\boldsymbol{X}, \boldsymbol{x}_{0}\right) \rightarrow \boldsymbol{\pi}_{k}\left(\boldsymbol{Y}, \boldsymbol{y}_{0}\right)=\boldsymbol{\pi}_{k}^{*}\left(\boldsymbol{Y}, \boldsymbol{y}_{0}\right)
$$

is an isomorphism of pro $^{*}$-Set 0 for $k=0$, an isomorphism of $p r o^{*}$-Grp for each $k=$ $1, \ldots, m-1$, and an epimorphism of pro*-Grp for $k=m$.

The next theorem is a full "*-analogue" of Theorem II.5.3.1 of [18].

Theorem 3.2. Let $m \in \mathbb{N}$, and let $\left(\boldsymbol{X}, \boldsymbol{x}_{0}\right)=\left(\left(X_{\lambda}, x_{\lambda}\right),\left[p_{\lambda \lambda^{\prime}}\right], \Lambda\right)$ and $\left(\boldsymbol{Y}, \boldsymbol{y}_{0}\right)=$ $\left(\left(Y_{\lambda}, y_{\lambda}\right),\left[q_{\lambda \lambda^{\prime}}\right], \Lambda\right)$ be inverse systems of pointed connected polyhedra (in $\left.\mathrm{HPol}_{0}\right)$ having the same cofinite index set $\Lambda$. Let a morphism $\boldsymbol{f}^{*}:\left(\boldsymbol{X}, \boldsymbol{x}_{0}\right) \rightarrow\left(\boldsymbol{Y}, \boldsymbol{y}_{0}\right)$ of pro*$\mathrm{HPol}_{0}$ admit a level representative $\left(1_{\Lambda},\left[f_{\lambda}^{n}\right]\right)$. Then $\boldsymbol{f}^{*}$ is an $m$-equivalence of pro*HTop $_{0}$ if and only if, for every $\lambda \in \Lambda$, there exist $a \lambda^{\prime} \geqslant \lambda$ and an $n \in \mathbb{N}$ such that, for every $n^{\prime} \geqslant n$, there are a pair of pointed connected $C W$-complexes $\left(P_{\lambda}^{n^{\prime}}, p_{\lambda}^{n^{\prime}}\right)$, $\left(Q_{\lambda}^{n^{\prime}}, q_{\lambda}^{n^{\prime}}\right)$, an m-equivalence $g_{\lambda}^{n^{\prime}}:\left(P_{\lambda}^{n^{\prime}}, p_{\lambda}^{n^{\prime}}\right) \rightarrow\left(Q_{\lambda}^{n^{\prime}}, q_{\lambda}^{n^{\prime}}\right)$ and maps $p_{\lambda}^{n^{\prime}}:\left(P_{\lambda}^{n^{\prime}}, p_{\lambda}^{n^{\prime}}\right) \rightarrow$ $\left(X_{\lambda}, x_{\lambda}\right), \quad p_{\lambda}^{\prime n^{\prime}}: \quad\left(X_{\lambda^{\prime}}, x_{\lambda^{\prime}}\right) \rightarrow\left(P_{\lambda}^{n^{\prime}}, p_{\lambda}^{n^{\prime}}\right), \quad q_{\lambda}^{n^{\prime}}:\left(Q_{\lambda}^{n^{\prime}}, q_{\lambda}^{n^{\prime}}\right) \rightarrow\left(Y_{\lambda}, y_{\lambda}\right) \quad$ and $q_{\lambda}^{\prime n^{\prime}}:$ $\left(Y_{\lambda^{\prime}}, y_{\lambda^{\prime}}\right) \rightarrow\left(Q_{\lambda}^{n^{\prime}}, q_{\lambda}^{n^{\prime}}\right)$ such that the following diagram commutes up to homotopy 
(in $\mathrm{HTop}_{0}$ ).

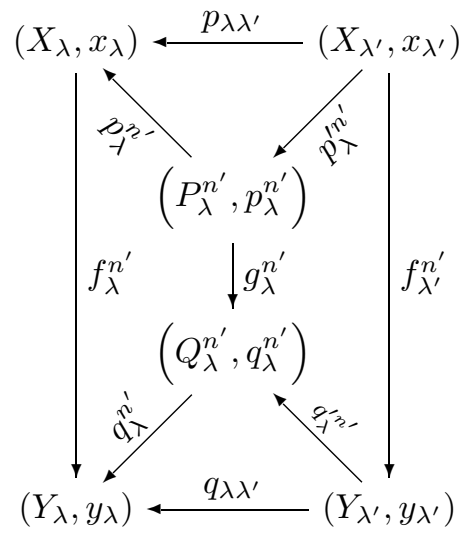

Proof. Let us first prove the sufficiency. Let $m \in \mathbb{N}$, and let $\left(1_{\Lambda},\left[f_{\lambda}^{n}\right]\right):\left(\boldsymbol{X}, \boldsymbol{x}_{0}\right) \rightarrow$ $\left(\boldsymbol{Y}, \boldsymbol{y}_{0}\right)$ be a level representative of $\boldsymbol{f}^{*}$. Suppose that, for every $\lambda \in \Lambda$, there exist a $\lambda^{\prime} \geqslant \lambda$ and an $n \in \mathbb{N}$ such that, for every $n^{\prime} \geqslant n$, there are a pair of pointed connected $C W$-complexes $\left(P_{\lambda}^{n^{\prime}}, p_{\lambda}^{n^{\prime}}\right),\left(Q_{\lambda}^{n^{\prime}}, q_{\lambda}^{n^{\prime}}\right)$, an $m$-equivalence $g_{\lambda}^{n^{\prime}}:\left(P_{\lambda}^{n^{\prime}}, p_{\lambda}^{n^{\prime}}\right) \rightarrow\left(Q_{\lambda}^{n^{\prime}}, q_{\lambda}^{n^{\prime}}\right)$, and maps

$$
\begin{aligned}
& p_{\lambda}^{n^{\prime}}:\left(P_{\lambda}^{n^{\prime}}, p_{\lambda}^{n^{\prime}}\right) \rightarrow\left(X_{\lambda}, x_{\lambda}\right), \quad p_{\lambda}^{\prime n^{\prime}}:\left(X_{\lambda^{\prime}}, x_{\lambda^{\prime}}\right) \rightarrow\left(P_{\lambda}^{n^{\prime}}, p_{\lambda}^{n^{\prime}}\right), \\
& q_{\lambda}^{n^{\prime}}:\left(Q_{\lambda}^{n^{\prime}}, q_{\lambda}^{n^{\prime}}\right) \rightarrow\left(Y_{\lambda}, y_{\lambda}\right), \quad q_{\lambda}^{\prime n^{\prime}}:\left(Y_{\lambda^{\prime}}, y_{\lambda^{\prime}}\right) \rightarrow\left(Q_{\lambda}^{n^{\prime}}, q_{\lambda}^{n^{\prime}}\right)
\end{aligned}
$$

such that diagram (1) commutes in HTop $_{0}$. We propose to prove that

$$
\boldsymbol{f}^{*}:\left(\boldsymbol{X}, \boldsymbol{x}_{0}\right) \rightarrow\left(\boldsymbol{Y}, \boldsymbol{y}_{0}\right)
$$

is an $m$-equivalence of $p r o^{*}-\mathrm{HTop}_{0}$. First, we are to verify that the induced homomorphisms

$$
\boldsymbol{f}_{k}^{*}: \boldsymbol{\pi}_{k}\left(\boldsymbol{X}, \boldsymbol{x}_{0}\right) \rightarrow \boldsymbol{\pi}_{k}\left(\boldsymbol{Y}, \boldsymbol{y}_{0}\right)
$$

are isomorphisms of pro $^{*}-$ Set $_{0}$, for $k=0$, and of pro ${ }^{*}-G r p$, for $k=1, \ldots, m-1$. It is sufficient to show that, for every $k=0, \ldots, m-1$, the level representative $\left(1_{\Lambda}, f_{\lambda k}^{n}\right) \in$ $\boldsymbol{f}_{k}^{*}$ (of $i n v^{*}-S_{0} t_{0} ; i n v^{*}-G r p$ ) fulfills the condition of Theorem 6.1 of [12] (a "pro*analogue" of the well-known Morita lemma). Let $\lambda \in \Lambda$ be an arbitrary index. By the theorem assumption, there exist a $\lambda^{\prime} \geqslant \lambda$ and an $n \in \mathbb{N}$ such that, for every $n^{\prime} \geqslant n$, diagram (1) commutes. Now, by operating the functor $\pi_{k}$ on that diagram, it yields the corresponding commutative diagram in pro ${ }^{*}$-Grp for $1 \leqslant k \leqslant m-1$ (in pro $^{*}$-Set 0 for $k=0$ ), which consists of the induced homomorphisms

$$
\begin{gathered}
p_{\lambda k}^{n^{\prime}}: \pi_{k}\left(P_{\lambda}^{n^{\prime}}, p_{\lambda}^{n^{\prime}}\right) \rightarrow \pi_{k}\left(X_{\lambda}, x_{\lambda}\right), p_{\lambda k}^{n^{\prime}}: \pi_{k}\left(X_{\lambda^{\prime}}, x_{\lambda^{\prime}}\right) \rightarrow \pi_{k}\left(P_{\lambda}^{n^{\prime}}, p_{\lambda}^{n^{\prime}}\right), \\
q_{\lambda k}^{n^{\prime}}: \pi_{k}\left(Q_{\lambda}^{n^{\prime}}, q_{\lambda}^{n^{\prime}}\right) \rightarrow \pi_{k}\left(Y_{\lambda}, y_{\lambda}\right), q_{\lambda k}^{\prime n^{\prime}}: \pi_{k}\left(Y_{\lambda^{\prime}}, y_{\lambda^{\prime}}\right) \rightarrow \pi_{k}\left(Q_{\lambda}^{n^{\prime}}, q_{\lambda}^{n^{\prime}}\right), \\
f_{\lambda k}^{n^{\prime}}: \pi_{k}\left(X_{\lambda}, x_{\lambda}\right) \rightarrow\left(Y_{\lambda}, y_{\lambda}\right), f_{\lambda^{\prime} k}^{n^{\prime}}: \pi_{k}\left(X_{\lambda^{\prime}}, x_{\lambda^{\prime}}\right) \rightarrow \pi_{k}\left(Y_{\lambda^{\prime}}, y_{\lambda^{\prime}}\right), \\
p_{\lambda \lambda^{\prime} k}: \pi_{k}\left(X_{\lambda^{\prime}}, x_{\lambda^{\prime}}\right) \rightarrow \pi_{k}\left(X_{\lambda}, x_{\lambda}\right), q_{\lambda \lambda^{\prime} k}: \pi_{k}\left(Y_{\lambda^{\prime}}, y_{\lambda^{\prime}}\right) \rightarrow \pi_{k}\left(Y_{\lambda}, y \lambda\right)
\end{gathered}
$$

and of the induced isomorphism

$$
g_{\lambda k}^{n^{\prime}}: \pi_{k}\left(P_{\lambda}^{n^{\prime}}, p_{\lambda}^{n^{\prime}}\right) \rightarrow \pi_{k}\left(Q_{\lambda}^{n^{\prime}}, q_{\lambda}^{n^{\prime}}\right) .
$$


Let us define, for every $n^{\prime} \geqslant n$, a homomorphism

$$
h_{\lambda}^{n^{\prime}}: \pi_{k}\left(Y_{\lambda^{\prime}}, y_{\lambda^{\prime}}\right) \rightarrow \pi_{k}\left(X_{\lambda}, x_{\lambda}\right)
$$

by putting

$$
h_{\lambda}^{n^{\prime}}=p_{\lambda k}^{n^{\prime}}\left(g_{\lambda k}^{n^{\prime}}\right)^{-1} q_{\lambda k}^{\prime n^{\prime}}
$$

Then

$$
f_{\lambda k}^{n^{\prime}} h_{\lambda}^{n^{\prime}}=f_{\lambda k}^{n^{\prime}} p_{\lambda k}^{n^{\prime}}\left(g_{\lambda k}^{n^{\prime}}\right)^{-1} q_{\lambda k}^{n^{\prime}}=q_{\lambda k}^{n^{\prime}} g_{\lambda k}^{n^{\prime}}\left(g_{\lambda k}^{n^{\prime}}\right)^{-1} q_{\lambda k}^{\prime n^{\prime}}=q_{\lambda k}^{n^{\prime}} q_{\lambda k}^{\prime n^{\prime}}=q_{\lambda \lambda^{\prime} k},
$$

and

$$
h_{\lambda}^{n^{\prime}} f_{\lambda^{\prime} k}^{n^{\prime}}=p_{\lambda k}^{n^{\prime}}\left(g_{\lambda k}^{n^{\prime}}\right)^{-1} q_{\lambda k}^{\prime n^{\prime}} f_{\lambda^{\prime} k}^{n^{\prime}}=p_{\lambda k}^{n^{\prime}}\left(g_{\lambda k}^{n^{\prime}}\right)^{-1} g_{\lambda k}^{n^{\prime}} p_{\lambda k}^{\prime n^{\prime}}=p_{\lambda k}^{n^{\prime}} p_{\lambda k}^{n^{\prime}}=p_{\lambda \lambda^{\prime} k} .
$$

According to Theorem 6.1 of $[\mathbf{1 2}]$, this implies that $\boldsymbol{f}_{k}^{*}=\left[\left(1_{\Lambda}, f_{\lambda k}^{n}\right)\right]$ is an isomorphism, for every $k=0, \ldots, m-1$. It remains to verify that

$$
\boldsymbol{f}_{m}^{*}: \boldsymbol{\pi}_{m}\left(\boldsymbol{X}, \boldsymbol{x}_{0}\right) \rightarrow \boldsymbol{\pi}_{m}\left(\boldsymbol{Y}, \boldsymbol{y}_{0}\right)
$$

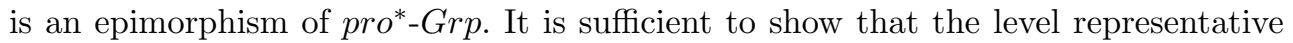
$\left(1_{\Lambda}, f_{\lambda m}^{n}\right) \in \boldsymbol{f}_{m}^{*}$ fulfills condition $(\mathrm{E}-\mathrm{Grp})$ of (4.5) of [9]. Let $\lambda \in \Lambda$ be an arbitrary index. By assumption, there exist a $\lambda^{\prime} \geqslant \lambda$ and an $n \in \mathbb{N}$ such that, for every $n^{\prime} \geqslant n$, diagram (1) commutes. Now, by operating the functor $\pi_{m}$ on that diagram, it yields the corresponding commutative diagram in pro*$^{*}-G r p$ which consists of the induced homomorphisms $p_{\lambda m}^{n^{\prime}}, p_{\lambda m}^{\prime n^{\prime}}, q_{\lambda m}^{n^{\prime}}, q_{\lambda m}^{\prime n^{\prime}}, f_{\lambda m}^{n^{\prime}}, f_{\lambda^{\prime} m}^{n^{\prime}}, p_{\lambda \lambda^{\prime} m}, q_{\lambda \lambda^{\prime} m}$, and epimorphism $g_{\lambda m}^{n^{\prime}}$. For a $y \in \operatorname{Im} q_{\lambda \lambda^{\prime} m}$, there exists a $y^{\prime} \in \pi_{m}\left(Y_{\lambda^{\prime}}, y_{\lambda^{\prime}}\right)$ such that $y=q_{\lambda \lambda^{\prime} m}\left(y^{\prime}\right)$. Thus, it holds that

$$
y=q_{\lambda m}^{n^{\prime}} q_{\lambda m}^{\prime n^{\prime}}\left(y^{\prime}\right)
$$

Since

$$
g_{\lambda m}^{n^{\prime}}: \pi_{m}\left(P_{\lambda}^{n^{\prime}}, p_{\lambda}^{n^{\prime}}\right) \rightarrow \pi_{m}\left(Q_{\lambda}^{n^{\prime}}, q_{\lambda}^{n^{\prime}}\right)
$$

is an epimorphism, for $q_{\lambda m}^{\prime n^{\prime}}\left(y^{\prime}\right) \in \pi_{m}\left(Q_{\lambda}^{n^{\prime}}, q_{\lambda}^{n^{\prime}}\right)$ there exists a $y^{\prime \prime} \in \pi_{m}\left(P_{\lambda}^{n^{\prime}}, p_{\lambda}^{n^{\prime}}\right)$ such that $q_{\lambda m}^{\prime n^{\prime}}\left(y^{\prime}\right)=g_{\lambda m}^{n^{\prime}}\left(y^{\prime \prime}\right)$. Therefore, (2) implies that

$$
y=q_{\lambda m}^{n^{\prime}} q_{\lambda m}^{\prime n^{\prime}}\left(y^{\prime}\right)=q_{\lambda m}^{n^{\prime}} g_{\lambda m}^{n^{\prime}}\left(y^{\prime \prime}\right) .
$$

Since $f_{\lambda m}^{n^{\prime}} p_{\lambda m}^{n^{\prime}}=q_{\lambda m}^{n^{\prime}} g_{\lambda m}^{n^{\prime}}$, it follows, by (3), that $y=f_{\lambda m}^{n^{\prime}} p_{\lambda m}^{n^{\prime}}\left(y^{\prime \prime}\right)$. Thus, we infer that $y \in \operatorname{Im} f_{\lambda m}^{n^{\prime}}$. Therefore, the $*$-morphism $\left(1_{\Lambda}, f_{\lambda m}^{n}\right) \in \boldsymbol{f}_{m}^{*}$ satisfies condition (E) (E-Grp) of (4.5) of [9], which implies that $\boldsymbol{f}_{m}^{*}$ is an epimorphism and, consequently, that $\boldsymbol{f}^{*}:(\boldsymbol{X}, *) \rightarrow(\boldsymbol{Y}, *)$ is an $m$-equivalence of pro $^{*}-\mathrm{HTop}_{0}$.

In order to prove the necessity part of Theorem 3.2 we first want to define some auxiliary objects and to establish several technical lemmata as follows.

Lemma 3.3. Let $m \in \mathbb{N}$, and let, for each $i=1, \ldots, m, p_{i}:\left(X_{i-1}, A_{i-1}, x_{i-1}\right) \rightarrow$ $\left(X_{i}, A_{i}, x_{i}\right)$ be a map of pointed pairs of $C W$-comlexes such that $X_{0}$ is connected and the induced homomorphism $\pi_{i}\left(p_{i}\right): \pi_{i}\left(X_{i-1}, A_{i-1}, x_{i-1}\right) \rightarrow \pi_{i}\left(X_{i}, A_{i}, x_{i}\right)$ is trivial. Then the composite map $p_{m} p_{m-1} \cdots p_{1}:\left(X_{0}, A_{0}, x_{0}\right) \rightarrow\left(X_{m}, A_{m}, x_{m}\right)$ factors through an $m$-connected pointed pair of $C W$-complexes $\left(Q, P, p_{0}\right)$ with $Q$ and $P$ connected. 
Proof. A polyhedral analogue of the above lemma is equivalent to Lemma II.4.2.3 of [18]. Further, by following the proof of that lemma, it is easy to verify that it remains valid if we replace a pointed polyhedral pair with a pointed pair of CW-complexes throughout.

Let us recall that, for a given map $f:\left(X, x_{0}\right) \rightarrow\left(Y, y_{0}\right)$ of $T o p_{0}$, the reduced mapping cylinder of $f$ is the space $\left(Z_{f}, z_{0}\right)$ obtained by attaching $X \times I$ to $Y$ by the map $(x, 1) \mapsto f(x), x \in X$, and $\left(x_{0}, t\right) \mapsto f\left(x_{0}\right)=y_{0}, t \in I$. Its base point is $z_{0}=\left\{p\left(\left(\left\{x_{0}\right\} \times I\right) \sqcup\left\{y_{0}\right\}\right)\right\}$, where $p:(X \times I) \sqcup Y \rightarrow Z_{f}$ denotes the corresponding projection. The restriction map

$$
j \equiv p \mid Y:\left(Y, y_{0}\right) \rightarrow\left(Z_{f}, z_{0}\right)\left(i \equiv p \mid(X \times 0):\left(X, x_{0}\right) \rightarrow\left(Z_{f}, z_{0}\right)\right)
$$

is an embedding which is called the canonical embedding onto upper (lower) base of $\left(Z_{f}, z_{0}\right)$. Further, the pointed homotopy class $[j]$ is an isomorphism of $\mathrm{HTop}_{0}$, and it holds that

$$
[j][f]=[i] .
$$

Let us recall that the wedge of a given family of pointed spaces $\left(X_{\alpha}, x_{\alpha}\right)_{\alpha \in A}$ is the pointed space

$$
\bigvee_{\alpha \in A}\left(X_{\alpha}, x_{\alpha}\right)=\left(\bigsqcup_{\alpha \in A}\left(X_{\alpha}, x_{\alpha}\right)\right) /\left(\bigsqcup_{\alpha \in A}\left\{x_{\alpha}\right\}\right)
$$

obtained by identifying all the base points $x_{\alpha}$ in the disjoint union of spaces $X_{\alpha}$, $\alpha \in A$, endowed with the weak topology. We denote the natural embedding of the pointed space $\left(X_{\alpha}, x_{\alpha}\right)$ into the wedge by

$$
\iota^{\alpha}:\left(X_{\alpha}, x_{\alpha}\right) \rightarrow \bigvee_{\alpha \in A}\left(X_{\alpha}, x_{\alpha}\right), \quad \text { for each } \alpha \in A .
$$

For a given family

$$
\left(f^{\alpha}:\left(X_{\alpha}, x_{\alpha}\right) \rightarrow\left(Y, y_{0}\right)\right)_{\alpha \in A}
$$

of maps, there exists the unique map

$$
f=\bigvee_{\alpha \in A} f^{\alpha}: \bigvee_{\alpha \in A}\left(X_{\alpha}, x_{\alpha}\right) \rightarrow\left(Y, y_{0}\right)
$$

such that $f \iota^{\alpha}=f^{\alpha}$, for every $\alpha \in A$. Further, for a given family

$$
\left(p^{\alpha}:\left(X_{\alpha}, x_{\alpha}\right) \rightarrow\left(X_{\alpha}^{\prime}, x_{\alpha}^{\prime}\right)\right)_{\alpha \in A}
$$

of maps, there exists the unique map

$$
p=\bigvee_{\alpha \in A} p^{\alpha}: \bigvee_{\alpha \in A}\left(X_{\alpha}, x_{\alpha}\right) \rightarrow \bigvee_{\alpha \in A}\left(X_{\alpha}^{\prime}, x_{\alpha}^{\prime}\right)
$$

of the wedges such that $p \iota^{\alpha}=\iota^{\prime \alpha} p^{\alpha}$, for every $\alpha \in A$. 
Let $\left(Z_{f}, z_{0}\right)$ be the reduced mapping cylinder of a map

$$
f: \bigvee_{\alpha \in A}\left(X_{\alpha}, x_{\alpha}\right) \rightarrow\left(Y, y_{0}\right)
$$

and let

$$
i: \bigvee_{\alpha \in A}\left(X_{\alpha}, x_{\alpha}\right) \rightarrow\left(Z_{f}, z_{0}\right)
$$

be the canonical embedding onto lower base of $\left(Z_{f}, z_{0}\right)$. Then, for every $\alpha \in A$, the composite map

$$
i^{\alpha} \equiv i \iota^{\alpha}:\left(X_{\alpha}, x_{\alpha}\right) \rightarrow\left(Z_{f}, z_{0}\right)
$$

is an embedding, which is called the canonical embedding of $\left(X_{\alpha}, x_{\alpha}\right)$ into lower base of $\left(Z_{f}, z_{0}\right)$. Consequently, $\left(X_{\alpha}, x_{\alpha}\right)$ can be regarded as the subspace of $\left(Z_{f}, z_{0}\right)$.

Lemma 3.4. Let $\left(f^{n}\right), f^{n}:\left(X_{n}, x_{n}\right) \rightarrow\left(Y, y_{0}\right)$, be a sequence of cellular mappings of pointed $C W$-complexes, where $Y$ is connected, and let $\left(Z_{f}, z_{0}\right)$ be the reduced mapping cylinder of the map

$$
f=\bigvee_{n \in \mathbb{N}} f^{n}: \bigvee_{n \in \mathbb{N}}\left(X_{n}, x_{n}\right) \rightarrow\left(Y, y_{0}\right) .
$$

If $j:\left(Y, y_{0}\right) \rightarrow\left(Z_{f}, z_{0}\right)$ and $i^{n}:\left(X_{n}, x_{n}\right) \rightarrow\left(Z_{f}, z_{0}\right), n \in \mathbb{N}$, are the canonical embeddings onto upper and into lower base of $\left(Z_{f}, z_{0}\right)$, respectively, then

(i) $\left(Z_{f}, z_{0}\right)$ is a connected $C W$-complex;

(ii) $\left(X_{n}, x_{n}\right)$ is the subcomplex of $\left(Z_{f}, z_{0}\right)$, for every $n \in \mathbb{N}$; and

(iii) $[j]\left[f^{n}\right]=\left[i^{n}\right]$ in $\mathrm{HTop}_{0}$, for every $n \in \mathbb{N}$.

Proof. Since $\bigvee_{n \in \mathbb{N}}\left(X_{n}, x_{n}\right)$ is a pointed $C W$-complex (Corollary 2.5.9 of [14]) and since $f: \bigvee_{n \in \mathbb{N}}\left(X_{n}, x_{n}\right) \rightarrow\left(Y, y_{0}\right)$ is a cellular mapping, it follows, by Corollaries 2.5.12 and 2.5.9 of [14], that $\left(Z_{f}, z_{0}\right)$ is a pointed $C W$-complex which admits a $C W$ decomposition having $\left(X_{n}, x_{n}\right)$ as its subcomplex, for every $n \in \mathbb{N}$. Since $Y$ is (pointed) homotopy equivalent (isomorphic in $\mathrm{HTop}_{0}$ ) to $Z_{f}$, it follows that $Z_{f}$ is connected. Finally, by (4), one obtains that

$$
[j]\left[f^{n}\right]=[j][f]\left[\iota^{n}\right]=[i]\left[\iota^{n}\right]=\left[i^{n}\right] .
$$

Proof of Theorem 3.2 (necessity). Let $m \in \mathbb{N}$, and let $\boldsymbol{f}^{*}:\left(\boldsymbol{X}, \boldsymbol{x}_{0}\right) \rightarrow\left(\boldsymbol{Y}, \boldsymbol{y}_{0}\right)$ be an $m$-equivalence of pro $^{*}-\mathrm{HTop}_{0}$ having a level representative $\left(1_{\Lambda},\left[f_{\lambda}^{n}\right]\right)$. Since the index set $\Lambda$ is cofinite, we may apply Lemma 1 of [8] to obtain, for every $\lambda \in \Lambda$, an integer $n_{\lambda} \in \mathbb{N}$ such that, for every $\lambda^{\prime} \leqslant \lambda$ and every $n \geqslant n_{\lambda}$, it holds that

$$
\left[f_{\lambda^{\prime}}^{n}\right]\left[p_{\lambda^{\prime} \lambda}\right]=\left[q_{\lambda^{\prime} \lambda}\right]\left[f_{\lambda}^{n}\right]
$$

in $\mathrm{HPol}_{0}$. Moreover, one can achieve that

$$
n_{\lambda^{\prime}} \leqslant n_{\lambda}
$$

for every $\lambda^{\prime} \leqslant \lambda$. For every $\lambda \in \Lambda$, let

$$
\left(X_{\lambda}^{0}, x_{\lambda}^{0}\right)=\bigvee_{n \in \mathbb{N}}\left(X_{\lambda}^{n}, x_{\lambda}^{n}\right)
$$


denote the wedge of the family (sequence) of pointed polyhedra $\left(X_{\lambda}^{n}, x_{\lambda}^{n}\right), n \in \mathbb{N}$,

$$
\left(X_{\lambda}^{n}, x_{\lambda}^{n}\right)= \begin{cases}\left(X_{\lambda}, x_{\lambda}\right), & n \geqslant n_{\lambda} \\ \left(\left\{x_{\lambda}\right\}, x_{\lambda}\right), & n<n_{\lambda} .\end{cases}
$$

Let $p_{\lambda \lambda^{\prime}}^{0}=\bigvee_{n \in \mathbb{N}} p_{\lambda \lambda^{\prime}}^{n}:\left(X_{\lambda^{\prime}}^{0}, x_{\lambda^{\prime}}^{0}\right) \rightarrow\left(X_{\lambda}^{0}, x_{\lambda}^{0}\right)$, where $p_{\lambda \lambda^{\prime}}^{n}=p_{\lambda \lambda^{\prime}} \mid X_{\lambda^{\prime}}^{n}:\left(X_{\lambda^{\prime}}^{n}, x_{\lambda^{\prime}}^{n}\right) \rightarrow$ $\left(X_{\lambda}^{n}, x_{\lambda}^{n}\right)$.

Notice that $\left(X_{\lambda}^{n}, x_{\lambda}^{n}\right)$ and $\left(Y_{\lambda}, y_{\lambda}\right)$, for all $\lambda \in \Lambda$ and $n \in \mathbb{N}$, are pointed $C W$ complexes with obvious $C W$-decompositions. For every $\lambda \in \Lambda$ and every $n \in \mathbb{N}$, let us choose a cellular map $f_{\lambda}^{n}:\left(X_{\lambda}, x_{\lambda}\right) \rightarrow\left(Y_{\lambda}, y_{\lambda}\right)$ of the pointed $C W$-complexes representing the (pointed) homotopy class $\left[f_{\lambda}^{n}\right]:\left(X_{\lambda}, x_{\lambda}\right) \rightarrow\left(Y_{\lambda}, y_{\lambda}\right)$ (cellular approximation theorem-Theorem 8.5. of [14]). Let $\left(Z_{\lambda}, z_{\lambda}\right)$ denote the reduced mapping cylinder of the map

$$
f_{\lambda}^{0}=\bigvee_{n \in \mathbb{N}}\left(f_{\lambda}^{n} \mid X_{\lambda}^{n}\right):\left(X_{\lambda}^{0}, x_{\lambda}^{0}\right)=\bigvee_{n \in \mathbb{N}}\left(X_{\lambda}^{n}, x_{\lambda}^{n}\right) \rightarrow\left(Y_{\lambda}, y_{\lambda}\right),
$$

for every $\lambda \in \Lambda$. For every $\lambda$ and every $n$, let

$$
i_{\lambda}^{n}:\left(X_{\lambda}^{n}, x_{\lambda}^{n}\right) \rightarrow\left(Z_{\lambda}, z_{\lambda}\right)\left(j_{\lambda}:\left(Y_{\lambda}, y_{\lambda}\right) \rightarrow\left(Z_{\lambda}, z_{\lambda}\right)\right)
$$

be the canonical embedding into lower (onto upper) base of $\left(Z_{\lambda}, z_{\lambda}\right)$, and let

$$
i_{\lambda}=\bigvee_{n \in \mathbb{N}} i_{\lambda}^{n}:\left(X_{\lambda}^{0}, x_{\lambda}^{0}\right) \rightarrow\left(Z_{\lambda}, z_{\lambda}\right)
$$

denote the canonical embedding onto lower base of $\left(Z_{\lambda}, z_{\lambda}\right)$.

Claim 3.5. $\left(Z_{\lambda}, z_{\lambda}\right)$ is a connected $C W$-complex having $\left(X_{\lambda}^{n}, x_{\lambda}^{n}\right)$ for its subcomplex, and it holds that $\left[j_{\lambda}\right]\left[f_{\lambda}^{n}\right]=\left[i_{\lambda}^{n}\right]$, for every $n \geqslant n_{\lambda}$ and every $\lambda \in \Lambda$.

Proof. Since, for every $\lambda \in \Lambda, f_{\lambda}^{n}$ is a cellular map, the restriction

$$
f_{\lambda}^{n} \mid X_{\lambda}^{n}= \begin{cases}f_{\lambda}^{n}:\left(X_{\lambda}, x_{\lambda}\right) \rightarrow\left(Y_{\lambda}, y_{\lambda}\right), & \text { for } n \geqslant n_{\lambda} \\ \text { const. }=*:\left(\left\{x_{\lambda}\right\}, x_{\lambda}\right) \rightarrow\left(Y_{\lambda}, y_{\lambda}\right), & \text { for } n<n_{\lambda},\end{cases}
$$

is a cellular map as well, for every $n \in \mathbb{N}$. Lemma 3.4 implies the first part of the claim. Further, since it holds $\left[j_{\lambda}\right]\left[f_{\lambda}^{n} \mid X_{\lambda}^{n}\right]=\left[i_{\lambda}^{n}\right]$ in $\mathrm{HTop}_{0}$, for all $\lambda$ and $n$, the claim follows by (8) and (4).

Claim 3.6. For every related pair $\lambda \leqslant \lambda^{\prime}$, there exists a map

$$
s_{\lambda \lambda^{\prime}}:\left(Z_{\lambda^{\prime}}, z_{\lambda^{\prime}}\right) \rightarrow\left(Z_{\lambda}, z_{\lambda}\right)
$$

such that the following diagram strictly commutes (in Top p $_{0}$.

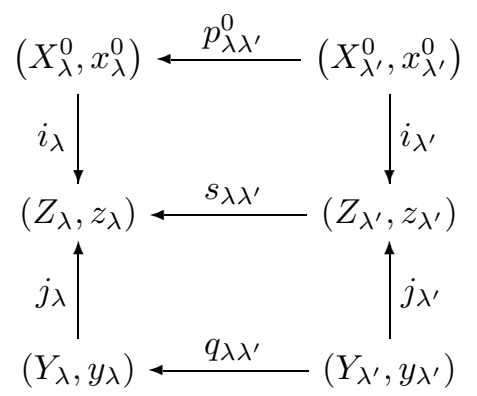


Therefore, the map $s_{\lambda \lambda^{\prime}}$ can be considered as a map

$$
s_{\lambda \lambda^{\prime}}:\left(Z_{\lambda^{\prime}}, X_{\lambda^{\prime}}^{0}, x_{\lambda^{\prime}}^{0}\right) \rightarrow\left(Z_{\lambda}, X_{\lambda}^{0}, x_{\lambda}^{0}\right)
$$

of pointed pairs of $C W$-complexes such that $s_{\lambda \lambda^{\prime}} \mid X_{\lambda^{\prime}}^{0}=p_{\lambda \lambda^{\prime}}^{0}$. Furthermore, whenever needed, we may consider $s_{\lambda \lambda^{\prime}}$ to be

$$
s_{\lambda \lambda^{\prime}}^{n}:\left(Z_{\lambda^{\prime}}, X_{\lambda^{\prime}}^{n}, x_{\lambda^{\prime}}^{n}\right) \rightarrow\left(Z_{\lambda}, X_{\lambda}^{n}, x_{\lambda}^{n}\right), n \geqslant n_{\lambda^{\prime}},
$$

the map of pointed pairs of $C W$-complexes such that

$$
s_{\lambda \lambda^{\prime}}^{n}\left|X_{\lambda^{\prime}}^{n}=s_{\lambda \lambda^{\prime}}\right| X_{\lambda^{\prime}}^{n}=p_{\lambda \lambda^{\prime}}:\left(X_{\lambda^{\prime}}, x_{\lambda^{\prime}}\right) \rightarrow\left(X_{\lambda}, x_{\lambda}\right),
$$

for every $n \geqslant n_{\lambda^{\prime}}$.

Proof. Notice that

$$
p_{\lambda \lambda^{\prime}}^{n}= \begin{cases}p_{\lambda \lambda^{\prime}}:\left(X_{\lambda^{\prime}}, x_{\lambda^{\prime}}\right) \rightarrow\left(X_{\lambda}, x_{\lambda}\right), & \text { for } n \geqslant n_{\lambda^{\prime}} \\ \text { const. }=*:\left(\left\{x_{\lambda^{\prime}}\right\}, x_{\lambda^{\prime}}\right) \rightarrow\left(X_{\lambda}, x_{\lambda}\right), & \text { for } n<n_{\lambda^{\prime}} .\end{cases}
$$

By $(5),(6),(7),(8)$, and (10), one obtains

$$
\left[f_{\lambda}^{n}\right]\left[p_{\lambda \lambda^{\prime}}^{n}\right]= \begin{cases}{\left[f_{\lambda}^{n}\right]\left[p_{\lambda \lambda^{\prime}}\right],} & \text { for } n \geqslant n_{\lambda^{\prime}} \\ *, & \text { for } n<n_{\lambda^{\prime}}\end{cases}
$$

and

$$
\left[q_{\lambda \lambda^{\prime}}\right]\left[f_{\lambda^{\prime}}^{n} \mid X_{\lambda^{\prime}}^{n}\right]= \begin{cases}{\left[q_{\lambda \lambda^{\prime}}\right]\left[f_{\lambda^{\prime}}^{n}\right],} & \text { for } n \geqslant n_{\lambda^{\prime}} \\ *, & \text { for } n<n_{\lambda^{\prime}}\end{cases}
$$

which imply that

$$
\left[f_{\lambda}^{n}\right]\left[p_{\lambda \lambda^{\prime}}^{n}\right]=\left[q_{\lambda \lambda^{\prime}}\right]\left[f_{\lambda^{\prime}}^{n} \mid X_{\lambda^{\prime}}^{n}\right], \quad \text { for all } \lambda \leqslant \lambda^{\prime} \text { and } n \in \mathbb{N} .
$$

Consequently, the following diagram homotopically commutes (in $\mathrm{HTop}_{0}$ ).

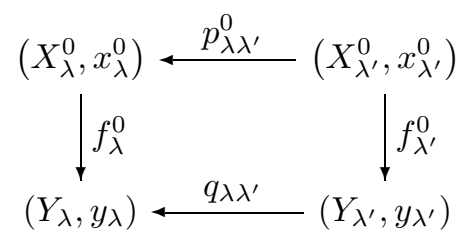

Then, the claim follows by Lemma II.5.1.3 of [18].

Claim 3.7. $\left(\boldsymbol{Z}, \boldsymbol{z}_{0}\right)=\left(\left(Z_{\lambda}, z_{\lambda}\right),\left[s_{\lambda \lambda^{\prime}}\right], \Lambda\right)$ is an inverse system in $\mathrm{HTop}_{0}$.

Proof. By Lemma II.5.1.3 of [18] and diagram (9), it follows that

$$
\left[s_{\lambda \lambda^{\prime}}\right]\left[s_{\lambda^{\prime} \lambda^{\prime \prime}}\right]\left[j_{\lambda^{\prime \prime}}\right]=\left[j_{\lambda}\right]\left[q_{\lambda \lambda^{\prime}}\right]\left[q_{\lambda^{\prime} \lambda^{\prime \prime}}\right]=\left[j_{\lambda}\right]\left[q_{\lambda \lambda^{\prime \prime}}\right]=\left[s_{\lambda \lambda^{\prime \prime}}\right]\left[j_{\lambda^{\prime \prime}}\right]
$$

in HTop $_{0}$. Since $j_{\lambda}:\left(Y_{\lambda}, y_{\lambda}\right) \rightarrow\left(Z_{\lambda}, z_{\lambda}\right)$ is the canonical embedding onto upper base of $\left(Z_{\lambda}, z_{\lambda}\right),\left[j_{\lambda}\right]$ is an isomorphism of $\mathrm{HTop}_{0}$ (a pointed homotopy equivalence), for every $\lambda \in \Lambda$. Consequently, for all $\lambda \leqslant \lambda^{\prime} \leqslant \lambda^{\prime \prime}$, it holds that

$$
\left[s_{\lambda \lambda^{\prime}}\right]\left[s_{\lambda^{\prime} \lambda^{\prime \prime}}\right]=\left[s_{\lambda \lambda^{\prime \prime}}\right] .
$$


Claim 3.8. $\left(1_{\Lambda},\left[\iota_{\lambda}^{n}\right]\right):\left(\boldsymbol{X}, \boldsymbol{x}_{0}\right) \rightarrow\left(\boldsymbol{Z}, \boldsymbol{z}_{0}\right)$ is a level $*$-morphism, where, for every $\lambda$ and every $n$, the map $\iota_{\lambda}^{n}:\left(X_{\lambda}, x_{\lambda}\right) \rightarrow\left(Z_{\lambda}, z_{\lambda}\right)$ is defined by the following rule:

$$
\iota_{\lambda}^{n}= \begin{cases}i_{\lambda}^{n}:\left(X_{\lambda}^{n}, x_{\lambda}^{n}\right) \rightarrow\left(Z_{\lambda}, z_{\lambda}\right), & \text { for } n \geqslant n_{\lambda} \\ \text { const. }=*, & \text { for } n<n_{\lambda} .\end{cases}
$$

Proof. By (9) and (10), for every related pair $\lambda \leqslant \lambda^{\prime}$ and every $n \geqslant n_{\lambda^{\prime}}$, one infers that

$$
\left[s_{\lambda \lambda^{\prime}}\right]\left[i_{\lambda^{\prime}}^{n}\right]=\left[s_{\lambda \lambda^{\prime}}\right]\left[i_{\lambda^{\prime}} \mid X_{\lambda^{\prime}}^{n}\right] \stackrel{(9)}{=}\left[i_{\lambda}\right]\left[p_{\lambda \lambda^{\prime}}^{0} \mid X_{\lambda^{\prime}}^{n}\right]=\left[i_{\lambda}\right]\left[p_{\lambda \lambda^{\prime}}^{n} \stackrel{(10)}{=}\left[i_{\lambda}^{n}\right]\left[p_{\lambda \lambda^{\prime}}\right],\right.
$$

which means that, for every $n \geqslant n_{\lambda^{\prime}}$, the following diagram commutes in $\mathrm{HTop}_{0}$ (moreover, strictly, i.e., in $T o p_{0}$ ).

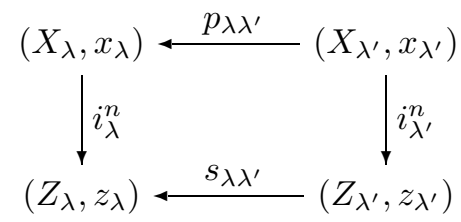

It follows that

$$
\left[s_{\lambda \lambda^{\prime}}\right]\left[\iota_{\lambda^{\prime}}^{n}\right]=\left[\iota_{\lambda}^{n}\right]\left[p_{\lambda \lambda^{\prime}}\right],
$$

for every pair $\lambda \leqslant \lambda^{\prime}$ and every $n \geqslant n_{\lambda^{\prime}}$.

Claim 3.9. $\boldsymbol{j}^{*}=\left[\left(1_{\Lambda},\left[j_{\lambda}^{n}\right]\right)\right]:\left(\boldsymbol{Y}, \boldsymbol{y}_{0}\right) \rightarrow\left(\boldsymbol{Z}, \boldsymbol{z}_{0}\right)$ is an isomorphism of pro* ${ }^{*} \mathrm{HTop}_{0}$, where $j_{\lambda}^{n}=j_{\lambda}$, for every $\lambda$ and all $n$.

Proof. By (9) (the lower rectangle of the diagram), we infer that

$$
\left(1_{\Lambda},\left[j_{\lambda}^{n}\right]\right):\left(\boldsymbol{Y}, \boldsymbol{y}_{0}\right) \rightarrow\left(\boldsymbol{Z}, \boldsymbol{z}_{0}\right)
$$

is the yielded $*$-morphism. Since $\left[j_{\lambda}^{n}\right]:\left(Y_{\lambda}, y_{\lambda}\right) \rightarrow\left(Z_{\lambda}, z_{\lambda}\right)$ is an isomorphism of $\mathrm{HTop}_{0}$, it follows, by Corollary 6.3. of [12], that $\boldsymbol{j}^{*}$ is an isomorphism.

Claim 3.10. $\iota^{*}=\left[\left(1_{\Lambda},\left[\iota_{\lambda}^{n}\right]\right)\right]$ is an m-equivalence of pro*-HPol${ }_{0}$.

Proof. By Claim 3.5 one infers that

$$
\left(1_{\Lambda},\left[j_{\lambda}^{n}\right]\right)\left(1_{\Lambda},\left[f_{\lambda}^{n}\right]\right) \sim\left(1_{\Lambda},\left[\iota_{\lambda}^{n}\right]\right)
$$

and, consequently, that

$$
\boldsymbol{j}^{*} \boldsymbol{f}^{*}=\iota^{*}=\left[\left(1_{\Lambda},\left[\iota_{\lambda}^{n}\right]\right)\right]
$$

in pro*$^{*}-\mathrm{HTop}_{0}$. Therefore,

$$
\boldsymbol{\pi}_{k}^{*}\left(\boldsymbol{j}^{*}\right) \boldsymbol{\pi}_{k}^{*}\left(\boldsymbol{f}^{*}\right)=\boldsymbol{\pi}_{k}^{*}\left(\iota^{*}\right)
$$

in pro $^{*}-G r p\left(\right.$ pro $^{*}-S_{0} t_{0}$, for $\left.k=0\right)$ holds. Since $\boldsymbol{j}^{*}$ is an isomorphism and $\boldsymbol{f}^{*}$ is an $m$-equivalence of pro $^{*}-\mathrm{HPol}_{0}$, one infers that $\boldsymbol{\iota}_{k}^{*}=\boldsymbol{\pi}_{k}^{*}\left(\iota^{*}\right)$ is an isomorphism of pro $^{*}$ Grp $\left(\right.$ pro $^{*}-$ Set $\left._{0}\right)$, for $k=1, \ldots, m-1(k=0)$, and an epimorphism of pro*-Grp, for $k=m$. Thus, $\iota^{*}$ is an $m$-equivalence of pro $^{*}-\mathrm{HPol}_{0}$. 
Claim 3.11. There exist functions $\varphi_{m}: \Lambda \rightarrow \Lambda$ and $\varepsilon_{m}: \Lambda \rightarrow \mathbb{N}$ such that, for every $\lambda \in \Lambda$ and every $n \geqslant \varepsilon_{m}(\lambda)$, it holds that

$$
\operatorname{Im} s_{\lambda \varphi_{m}(\lambda), m} \subseteq \operatorname{Im} \iota_{\lambda, m}^{n} .
$$

There exist functions $\varphi_{k}: \Lambda \rightarrow \Lambda$ and $\varepsilon_{k}: \Lambda \rightarrow \mathbb{N}$ such that, for every $\lambda \in \Lambda$ and every $n \geqslant \varepsilon_{k}(\lambda)$, there exists a homomorphism

$$
h_{\lambda k}^{n}: \pi_{k}\left(Z_{\varphi_{k}(\lambda)}, z_{\varphi_{k}(\lambda)}\right) \rightarrow \pi_{k}\left(X_{\lambda}, x_{\lambda}\right)
$$

that makes the following diagram commutative (in $\operatorname{Set}_{0}$, Grp).

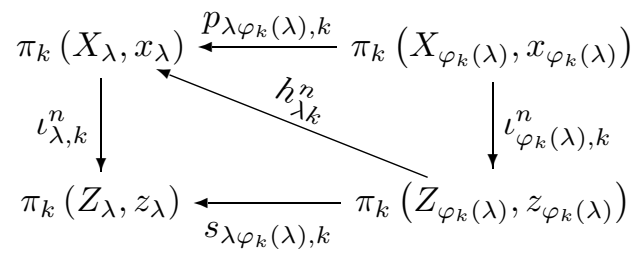

Proof. Since $\boldsymbol{\iota}_{m}^{*}: \boldsymbol{\pi}_{m}\left(\boldsymbol{X}, \boldsymbol{x}_{0}\right) \rightarrow \boldsymbol{\pi}_{m}\left(\boldsymbol{Z}, \boldsymbol{z}_{0}\right)$ is an epimorphism, and since, for every $k=0, \ldots, m-1, \iota_{k}^{*}: \boldsymbol{\pi}_{k}\left(\boldsymbol{X}, \boldsymbol{x}_{0}\right) \rightarrow \boldsymbol{\pi}_{k}\left(\boldsymbol{Z}, \boldsymbol{z}_{0}\right)$ is an isomorphism, there exist, according to condition (E-Grp) of 4.5 of [9] and Theorem 6.1 of [12], the functions $\varphi_{k}$ and $\varepsilon_{k}$ and homomorphisms $h_{\lambda k}^{n}$ such that (13) and (14) hold.

Claim 3.12. For every related pair $\lambda \leqslant \lambda^{\prime}$, for every $n \geqslant n_{\lambda^{\prime}}$ and for every $k \in \mathbb{N}$, the following diagram has exact columns and commutes in Grp (Set $\left.{ }_{0}\right)$.

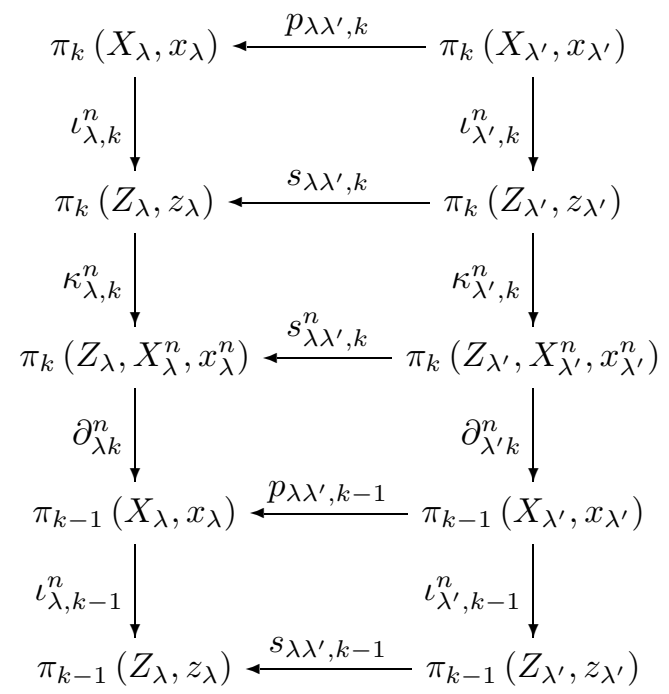

where $\kappa_{\lambda}^{n}:\left(Z_{\lambda}, z_{\lambda}, z_{\lambda}\right) \hookrightarrow\left(Z_{\lambda}, X_{\lambda}^{n}, x_{\lambda}^{n}\right)$ denotes the inclusion map and

$$
\partial_{\lambda k}^{n} \equiv \partial_{k}\left(Z_{\lambda}, X_{\lambda}^{n}, x_{\lambda}^{n}\right):\left(Z_{\lambda}, X_{\lambda}^{n}, x_{\lambda}^{n}\right) \rightarrow\left(X_{\lambda}, x_{\lambda}\right)=\left(X_{\lambda}^{n}, x_{\lambda}^{n}\right)
$$

is the boundary homomorphism (pointed function).

Proof. By (11) and (7), for every $\lambda \in \Lambda$ and every $n \geqslant n_{\lambda}$, the map

$$
\iota_{\lambda}^{n}:\left(X_{\lambda}, x_{\lambda}\right)=\left(X_{\lambda}^{n}, x_{\lambda}^{n}\right) \hookrightarrow\left(Z_{\lambda}, z_{\lambda}\right)
$$

is an inclusion. Therefore, it induces a long exact sequence of homotopy groups, i.e., 
for every $k \in \mathbb{N}$, every $\lambda \in \Lambda$, and every $n \geqslant n_{\lambda}$, the exact sequence in $\operatorname{Grp}\left(\operatorname{Set}_{0}\right)$ :

$$
\begin{aligned}
\pi_{k}\left(X_{\lambda}, x_{\lambda}\right) & \stackrel{\iota_{\lambda, k}^{n}}{\rightarrow} \pi_{k}\left(Z_{\lambda}, z_{\lambda}\right) \\
& \stackrel{\kappa_{\lambda \rightarrow k}^{n}}{\rightarrow} \pi_{k}\left(Z_{\lambda}, X_{\lambda}^{n}, x_{\lambda}^{n}\right) \stackrel{\partial_{\lambda, k}^{n}}{\rightarrow} \pi_{k-1}\left(X_{\lambda}, x_{\lambda}\right) \stackrel{i_{\lambda, k-1}^{n} \rightarrow}{\rightarrow} \pi_{k-1}\left(Z_{\lambda}, z_{\lambda}\right) .
\end{aligned}
$$

Since, by Claim 3.6, we may consider $s_{\lambda \lambda^{\prime}}$,

$$
s_{\lambda \lambda^{\prime}}^{n}:\left(Z_{\lambda^{\prime}}, X_{\lambda^{\prime}}^{n}, x_{\lambda^{\prime}}^{n}\right) \rightarrow\left(Z_{\lambda}, X_{\lambda}^{n}, x_{\lambda}^{n}\right), n \geqslant n_{\lambda^{\prime}},
$$

as the map of pointed pairs of $C W$-complexes that induces the mapping of exact sequences (16). Therefore, for every $n \geqslant n_{\lambda^{\prime}}\left((6)\right.$ also implies that $\left.n \geqslant n_{\lambda}\right)$ and every $k \in \mathbb{N}$, the diagram (15) commutes in $\operatorname{Grp}\left(\operatorname{Set}_{0}\right)$.

Claim 3.13. Given a $\lambda \in \Lambda$, there exists a finite sequence of indices $\lambda_{k} \in \Lambda, k=$ $0, \ldots, m$, such that

$$
\lambda_{m}=\lambda \leqslant \lambda_{m-1} \leqslant \cdots \leqslant \lambda_{k} \leqslant \lambda_{k-1} \leqslant \cdots \leqslant \lambda_{0},
$$

and there exists a finite sequence of integers $n^{(k)}, k=1, \ldots, m$, such that

$$
o=s_{\lambda_{k} \lambda_{k-1, k}}^{n}=\pi_{k}\left(s_{\lambda_{k} \lambda_{k-1}}^{n}\right): \pi_{k}\left(Z_{\lambda_{k-1}}, X_{\lambda_{k-1}}^{n}, x_{\lambda_{k-1}}^{n}\right) \rightarrow \pi_{k}\left(Z_{\lambda_{k}}, X_{\lambda_{k}}^{n}, x_{\lambda_{k}}^{n}\right)
$$

is the trivial homomorphism, for every $n \geqslant n^{(k)}$ and every $k=1, \ldots, m$.

Proof. Put $\lambda_{m}=\lambda$. We use Claim 3.11 to define

$$
\begin{aligned}
& \lambda_{m}^{\prime}=\varphi_{m}\left(\lambda_{m}\right), \quad \lambda_{m-1}=\varphi_{m-1}\left(\lambda_{m}^{\prime}\right), \quad \text { and } \\
& n^{(m)}=\max \left\{\varepsilon_{m}\left(\lambda_{m}\right), \varepsilon_{m-1}\left(\lambda_{m}^{\prime}\right), n_{\lambda_{m-1}}\right\} .
\end{aligned}
$$

Then, by (14) and (15), for every $n \geqslant n^{(m)}$, the following diagram, having exact columns, commutes.

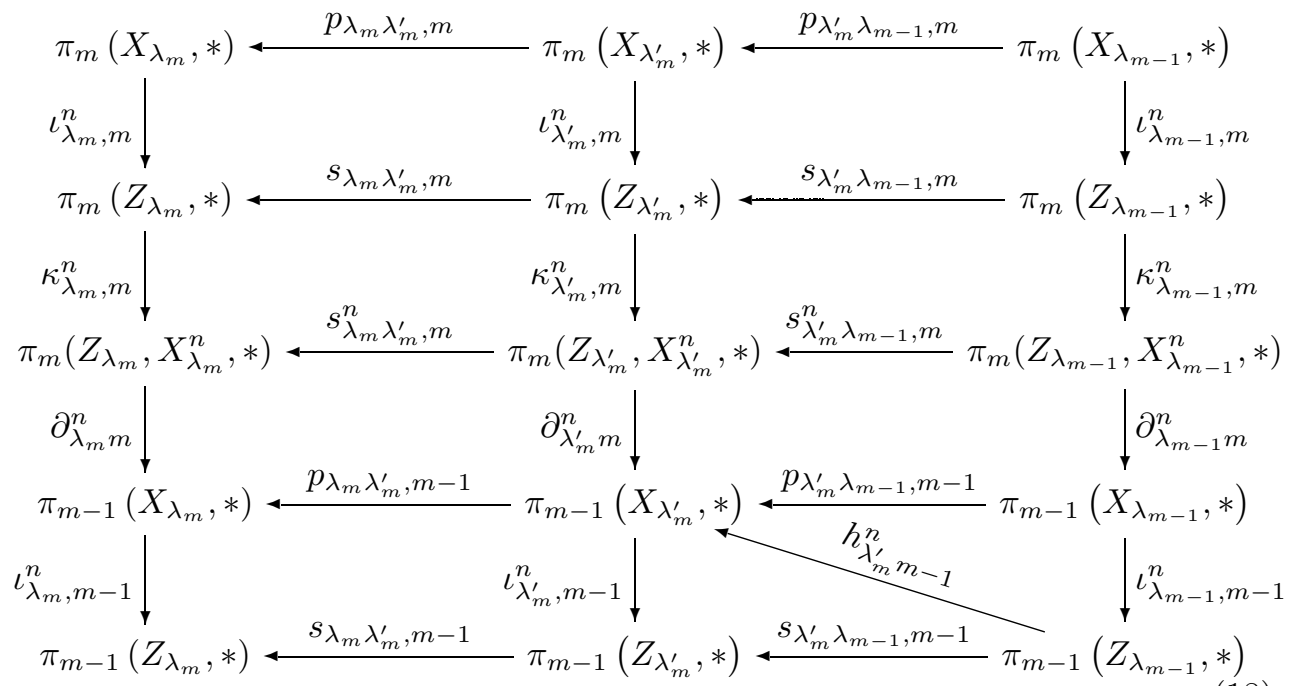

Let us check, by chasing diagram (18), that

$$
s_{\lambda_{m} \lambda_{m-1, m}}^{n}: \pi_{m}\left(Z_{\lambda_{m-1}}, X_{\lambda_{m-1}}^{n}, x_{\lambda_{m-1}}^{n}\right) \rightarrow \pi_{m}\left(Z_{\lambda_{m}}, X_{\lambda_{m}}^{n}, x_{\lambda_{m}}^{n}\right)
$$


is trivial, for every $n \geqslant n^{(m)}$. Let $n \geqslant n^{(m)}$, and let $x \in \pi_{m}\left(Z_{\lambda_{m-1}}, X_{\lambda_{m-1}}^{n}, x_{\lambda_{m-1}}^{n}\right) \quad$ (the 3rd row and the 3rd column of diagram (18)). Since the 3 rd column of diagram (18) is exact, it follows that

$$
\iota_{\lambda_{m-1}, m-1}^{n} \partial_{\lambda_{m-1} m}^{n}(x)=0,
$$

and, consequently,

$$
h_{\lambda_{m}^{\prime} m-1}^{n} \iota_{\lambda_{m-1}, m-1}^{n} \partial_{\lambda_{m-1} m}^{n}(x)=0 .
$$

Then, by commutativity of diagram (18), one infers that

$$
\partial_{\lambda_{m}^{\prime} m}^{n} s_{\lambda_{m}^{\prime} \lambda_{m-1}, m}^{n}(x)=0
$$

and, consequently, that

$$
s_{\lambda_{m}^{\prime} \lambda_{m-1}, m}^{n}(x) \in \operatorname{ker} \partial_{\lambda_{m}^{\prime}, m}^{n} .
$$

The exactness of the 2 nd column of diagram (18) implies that

$$
s_{\lambda_{m}^{\prime} \lambda_{m-1}, m}^{n}(x) \in \operatorname{Im} \kappa_{\lambda_{m}^{\prime}, m}^{n} .
$$

Therefore, there exists an

$$
x^{\prime} \in \pi_{m}\left(Z_{\lambda_{m}^{\prime}}, z_{\lambda_{m}^{\prime}}\right) \quad \text { (the 2nd row and the 2nd column of (18)) }
$$

such that

$$
\kappa_{\lambda_{m}^{\prime}, m}^{n}\left(x^{\prime}\right)=s_{\lambda_{m}^{\prime} \lambda_{m-1}, m}^{n}(x) .
$$

Since $n \geqslant \varepsilon_{m}\left(\lambda_{m}\right)$, it follows, by (13), that

$$
s_{\lambda_{m} \lambda_{m}^{\prime}, m}\left(x^{\prime}\right) \in \operatorname{Im} \iota_{\lambda_{m}, m}^{n} .
$$

Then, by the exactness of the 1st column of (18), it follows that

$$
\kappa_{\lambda_{m}, m}^{n} s_{\lambda_{m} \lambda_{m}^{\prime}, m}\left(x^{\prime}\right)=0 .
$$

The commutativity of diagram (18) implies that

$$
s_{\lambda_{m} \lambda_{m}^{\prime}, m}^{n} \kappa_{\lambda_{m}^{\prime}, m}^{n}\left(x^{\prime}\right)=0 .
$$

Finally, by (19), it follows that

$$
s_{\lambda_{m} \lambda_{m}^{\prime}, m}^{n} s_{\lambda_{m}^{\prime} \lambda_{m-1}, m}^{n}(x)=0 .
$$

Therefore, the homomorphism $s_{\lambda_{m} \lambda_{m-1, m}}^{n}$ is trivial. Hereby, we have proved (17) in the case $m=1$. If $m>1$, suppose that, for an $l \in \mathbb{N}, l \leqslant m-1$, we have already proven the existence of indices $\lambda_{k} \in \Lambda, k=l, \ldots, m-1, \lambda_{l} \geqslant \cdots \geqslant \lambda_{m-1} \geqslant \lambda_{m}$, and integers $n^{(k)}, k=l+1, \ldots, m$, such that, for every $k \in\{l+1, \ldots, m\}$ and every $n \geqslant n^{(k)}$,

$$
o=s_{\lambda_{k} \lambda_{k-1, k}}^{n}: \pi_{k}\left(Z_{\lambda_{k-1}}, X_{\lambda_{k-1}}^{n}, x_{\lambda_{k-1}}^{n}\right) \rightarrow \pi_{k}\left(Z_{\lambda_{k}}, X_{\lambda_{k}}^{n}, X_{\lambda_{k}}^{n}\right)
$$

is the trivial homomorphism. In order to prove (17), it is sufficient to prove the existence of an index $\lambda_{l-1}, \lambda_{l-1} \geqslant \lambda_{l}$ and an integer $n^{(l)}$ such that

$$
s_{\lambda_{l} \lambda_{l-1, l}}^{n}: \pi_{l}\left(Z_{\lambda_{l-1}}, X_{\lambda_{l-1}}^{n}, x_{\lambda_{l-1}}^{n}\right) \rightarrow \pi_{l}\left(Z_{\lambda_{l}}, X_{\lambda_{l}}^{n}, x_{\lambda_{l}}^{n}\right)
$$

is trivial, for every $n \geqslant n^{(l)}$. Let us choose $\lambda_{l}^{\prime}=\varphi_{l}\left(\lambda_{l}\right)$ and $\lambda_{l-1}=\varphi_{l-1}\left(\lambda_{l}^{\prime}\right)$, and let $n^{(l)}=\max \left\{\varepsilon_{l}\left(\lambda_{l}\right), \varepsilon_{l-1}\left(\lambda_{l}^{\prime}\right), n_{\lambda_{l-1}}\right\}$. Then, by (14) and (15), for every $n \geqslant n^{(l)}$, the 
following diagram, having exact columns, is commutative.

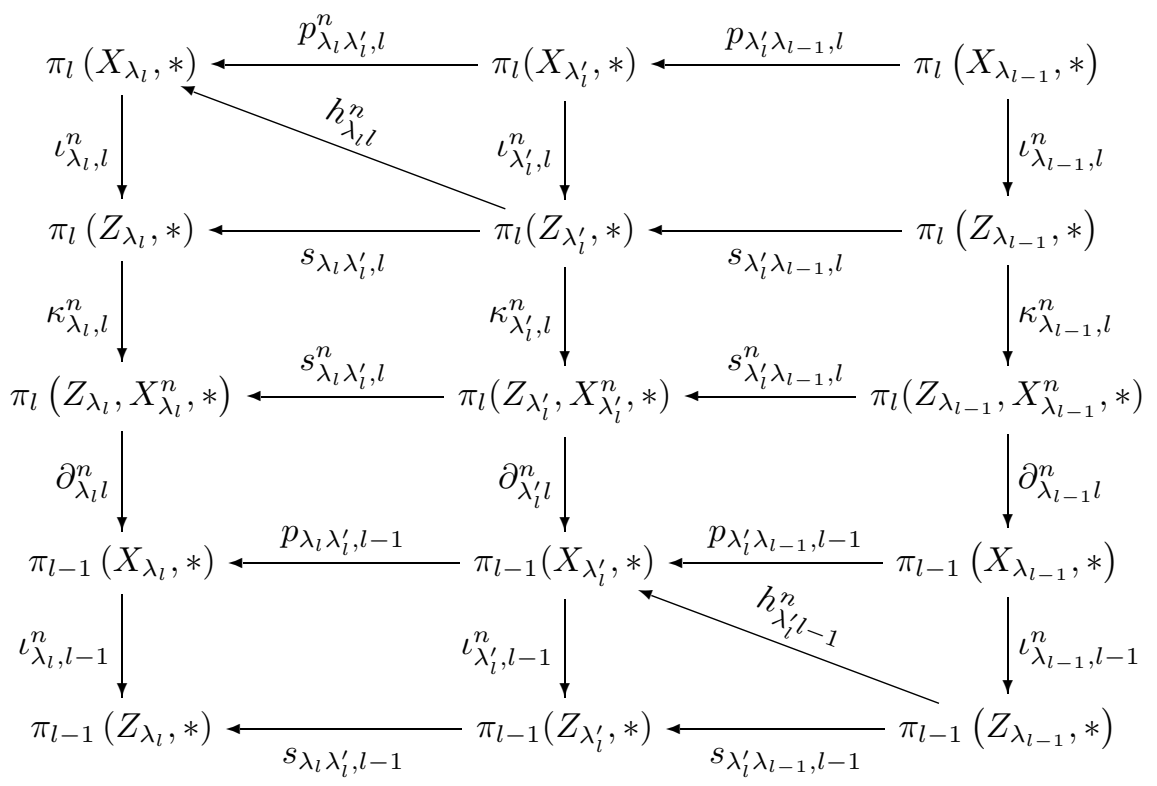

Let $n \geqslant n^{(l)}$ and let

$x \in \pi_{l}\left(Z_{\lambda_{l-1}}, X_{\lambda_{l-1}}^{n}, x_{\lambda_{l-1}}^{n}\right) \quad$ (the 3rd row and the 3rd column of diagram (20)).

Since the 3rd column of diagram (20) is exact, it follows that

$$
\iota_{\lambda_{l-1}, l-1}^{n} \partial_{\lambda_{l-1} l}^{n}(x)=0,
$$

and, consequently, that

$$
h_{\lambda_{l}^{\prime} l-1}^{n} \iota_{\lambda_{l-1}, l-1}^{n} \partial_{\lambda_{l-1} l}^{n}(x)=0 .
$$

Then, by commutativity of diagram (20), one infers that

$$
\partial_{\lambda_{l}^{\prime} l}^{n} s_{\lambda_{l}^{\prime} \lambda_{l-1}, l}^{n}(x)=0
$$

which implies that

$$
s_{\lambda_{l}^{\prime} \lambda_{l-1}, l}^{n}(x) \in \operatorname{ker} \partial_{\lambda_{l}^{\prime}, l}^{n} .
$$

The exactness of the 2 nd column of diagram (20) implies that

$$
s_{\lambda_{l}^{\prime} \lambda_{l-1}, l}^{n}(x) \in \operatorname{Im} \kappa_{\lambda_{l}^{\prime}, l}^{n} \text {. }
$$

Therefore, there exists an $x^{\prime} \in \pi_{l}\left(Z_{\lambda_{l}^{\prime}}, z_{\lambda_{l}^{\prime}}\right)$ (the 2nd row and the 2nd column of (20)) such that

$$
\kappa_{\lambda_{l}^{\prime}, l}^{n}\left(x^{\prime}\right)=s_{\lambda_{l}^{\prime} \lambda_{l-1}, l}^{n}(x) .
$$

By the exactness of the 1st column of (20), it follows that

$$
\kappa_{\lambda_{l}, l}^{n} \iota_{\lambda_{l}, l}^{n} h_{\lambda_{l} l}^{n}\left(x^{\prime}\right)=0 .
$$


Now, since diagram (20) commutes, it follows that

$$
\kappa_{\lambda_{l}, l}^{n} s_{\lambda_{l} \lambda_{l}^{\prime}, l}\left(x^{\prime}\right)=0
$$

which implies that

$$
s_{\lambda_{l} \lambda_{l}^{\prime}, l}^{n} \kappa_{\lambda_{l}^{\prime}, l}^{n}\left(x^{\prime}\right)=0
$$

This, along with (21), implies that $s_{\lambda_{l} \lambda_{l}^{\prime}, l^{n}}^{n} s_{\lambda_{l}^{\prime} \lambda_{l-1}, l}^{n}(x)=0$. Therefore, we may conclude that $s_{\lambda_{l} \lambda_{l-1}, l}^{n}=0$. This completes the proof of the claim.

Proof of necessity (continuation). For an arbitrary $\lambda$, let $\lambda_{m}=\lambda \leqslant \lambda_{m-1} \leqslant \cdots$ $\leqslant \lambda_{k} \leqslant \lambda_{k-1} \leqslant \cdots \leqslant \lambda^{\prime}=\lambda_{0}$ and $n^{(1)}, \ldots, n^{(m)}$ be as in Claim 3.13. Then, for every $n^{\prime} \geqslant n=\max \left\{n^{(k)} \mid k=1, \ldots, m\right\}$, the homomorphism $s_{\lambda_{k} \lambda_{k-1, k}}^{n^{\prime}}$ is trivial, $k=$ $1, \ldots, m$. Consequently, by Lemma 3.3 , the map

$$
s_{\lambda \lambda^{\prime}}^{n^{\prime}}=s_{\lambda_{m} \lambda_{m-1}}^{n^{\prime}} \cdots s_{\lambda_{k} \lambda_{k-1}}^{n^{\prime}} \cdots s_{\lambda_{1} \lambda_{0}}^{n^{\prime}}:\left(Z_{\lambda^{\prime}}, X_{\lambda^{\prime}}^{n^{\prime}}, x_{\lambda^{\prime}}^{n^{\prime}}\right) \rightarrow\left(Z_{\lambda}, X_{\lambda}^{n^{\prime}}, X_{\lambda}^{n^{\prime}}\right)
$$

factors through an $m$-connected pointed pair of $C W$-complexes $\left(Q_{\lambda}^{n^{\prime}}, P_{\lambda}^{n^{\prime}}, p_{\lambda}^{n^{\prime}}\right)$, such that $Q_{\lambda}^{n^{\prime}}$ and $P_{\lambda}^{n^{\prime}}$ are connected. Then there exist maps

$$
\begin{aligned}
& r_{\lambda}^{n^{\prime}}:\left(Q_{\lambda}^{n^{\prime}}, P_{\lambda}^{n^{\prime}}, p_{\lambda}^{n^{\prime}}\right) \rightarrow\left(Z_{\lambda}, X_{\lambda}^{n^{\prime}}, x_{\lambda}^{n^{\prime}}\right), \quad \text { and } \\
& r_{\lambda}^{\prime n^{\prime}}:\left(Z_{\lambda^{\prime}}, X_{\lambda^{\prime}}^{n^{\prime}}, x_{\lambda^{\prime}}^{n^{\prime}}\right) \rightarrow\left(Q_{\lambda}^{n^{\prime}}, P_{\lambda}^{n^{\prime}}, p_{\lambda}^{n^{\prime}}\right)
\end{aligned}
$$

such that $s_{\lambda \lambda^{\prime}}^{n^{\prime}}=r_{\lambda}^{n^{\prime}} r_{\lambda}^{\prime n^{\prime}}$.

Let $p_{\lambda}^{n^{\prime}}:\left(P_{\lambda}^{n^{\prime}}, p_{\lambda}^{n^{\prime}}\right) \rightarrow\left(X_{\lambda}^{n^{\prime}}, x_{\lambda}^{n^{\prime}}\right)$ and $p_{\lambda}^{n^{\prime}}:\left(X_{\lambda^{\prime}}^{n^{\prime}}, X_{\lambda^{\prime}}^{n^{\prime}}\right) \rightarrow\left(P_{\lambda}^{n^{\prime}}, p_{\lambda}^{n^{\prime}}\right)$ be the restriction maps $p_{\lambda}^{n^{\prime}} \equiv r_{\lambda}^{n^{\prime}} \mid P_{\lambda}^{n^{\prime}}$ and $p_{\lambda}^{\prime n^{\prime}} \equiv r_{\lambda}^{\prime n^{\prime}} \mid X_{\lambda^{\prime}}^{n^{\prime}}$, respectively, and let $g_{\lambda}^{n^{\prime}}:\left(P_{\lambda}^{n^{\prime}}, p_{\lambda}^{n^{\prime}}\right) \hookrightarrow$ $\left(Q_{\lambda}^{n^{\prime}}, q_{\lambda}^{n^{\prime}}\right)$ be the inclusion map. Notice that $g_{\lambda}^{n^{\prime}}$ is an $m$-equivalence (since $\left(Q_{\lambda}^{n^{\prime}}, P_{\lambda}^{n^{\prime}}, p_{\lambda}^{n^{\prime}}\right)$ is $m$-connected). Then the following diagram homotopically commutes (moreover, strictly, in $T o p_{0}$ ).

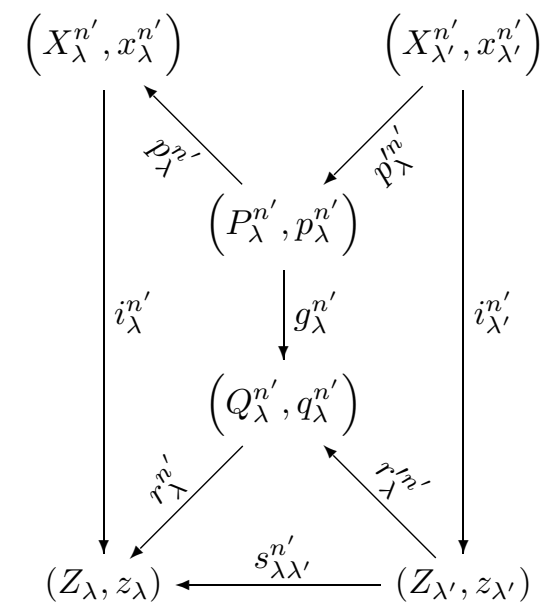

Since $n^{\prime} \geqslant n_{\lambda^{\prime}}\left(\geqslant n_{\lambda}\right)$, one infers, by (7) and Claim 3.6, that the following diagram 
homotopically commutes (moreover, strictly, in $T o p_{0}$ ).

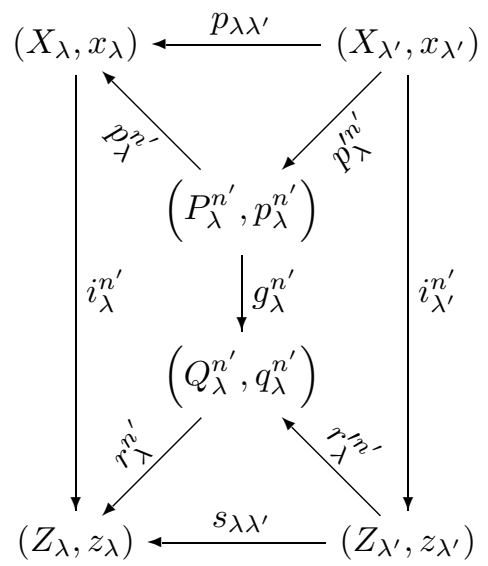

By using the previous diagram, equality (11) and the lower rectangle of diagram (13), one concludes that the following diagram commutes up to homotopy (in $\mathrm{HTop}_{0}$ ).

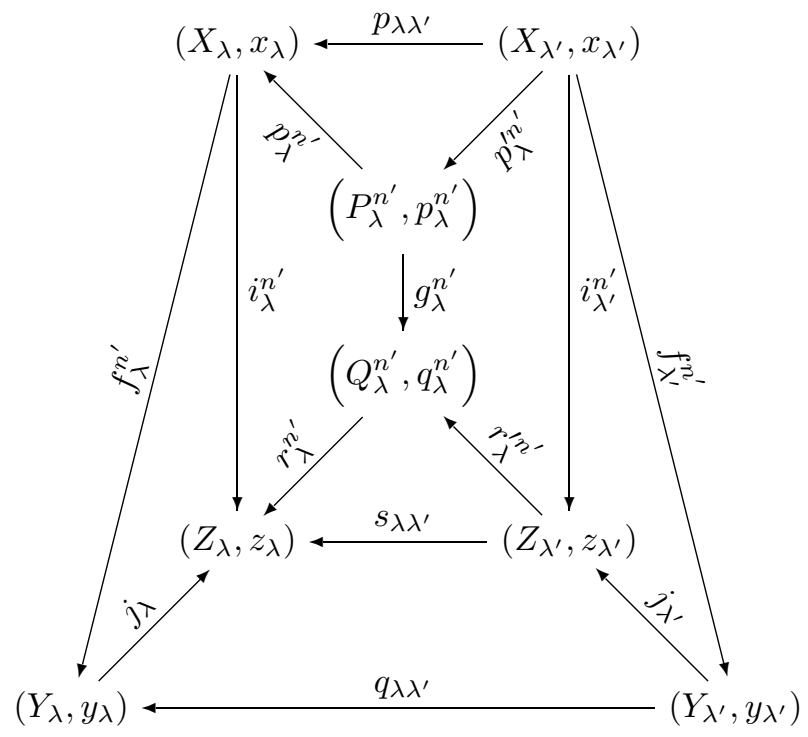

Finally, let us put $\left[q_{\lambda}^{n^{\prime}}\right]=\left[j_{\lambda}\right]^{-1}\left[r_{\lambda}^{n^{\prime}}\right]:\left(Q_{\lambda}^{n^{\prime}}, q_{\lambda}^{n^{\prime}}\right) \rightarrow\left(Y_{\lambda}, y_{\lambda}\right)$ and

$$
\left[q_{\lambda}^{\prime n^{\prime}}\right]=\left[r_{\lambda}^{\prime n^{\prime}}\right]\left[j_{\lambda^{\prime}}\right]:\left(Y_{\lambda^{\prime}}, y_{\lambda^{\prime}}\right) \rightarrow\left(Q_{\lambda}^{n^{\prime}}, q_{\lambda}^{n^{\prime}}\right)
$$

Then, by (22), it follows that diagram (1) commutes up to homotopy (in HTop $_{0}$ ), which, finally, completes the proof of necessity part of Theorem 3.2 as well.

Theorem 3.14. Let $\left(\boldsymbol{X}, \boldsymbol{x}_{0}\right)$ and $\left(\boldsymbol{Y}, \boldsymbol{y}_{0}\right)$ be inverse systems of pointed connected polyhedra (in $\mathrm{HPol}_{0}$ ) over the same cofinite index set $\Lambda$. Let $m \in \mathbb{N}$, and let $\boldsymbol{f}^{*}$ : $\left(\boldsymbol{X}, \boldsymbol{x}_{0}\right) \rightarrow\left(\boldsymbol{Y}, \boldsymbol{y}_{0}\right)$ be an m-equivalence of pro*-HPol ${ }_{0} \subseteq$ pro* $^{*}-\mathrm{HTop}_{0}$ which admits a level representative $\left(1_{\Lambda},\left[f_{\lambda}^{n}\right]\right)$. Then, for every $\lambda \in \Lambda$, there exist $a \lambda^{\prime} \geqslant \lambda$ and an $n \in \mathbb{N}$ such that, for every $n^{\prime} \geqslant n$, the following conditions are satisfied: 
(i) For every map $s:(R, r) \rightarrow\left(Y_{\lambda^{\prime}}, y_{\lambda^{\prime}}\right)$ of pointed connected polyhedra, with $\operatorname{dim} R \leqslant m$, there exists a map $k^{n^{\prime}}:(R, r) \rightarrow\left(X_{\lambda}, x_{\lambda}\right)$ such that the following diagram commutes up to homotopy (in $\mathrm{HPol}_{0}$ ).

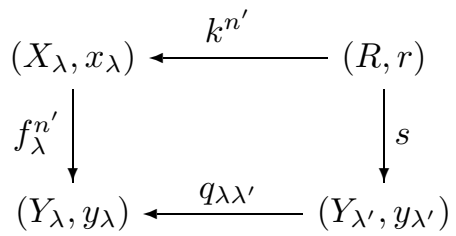

(ii) If $k, k^{\prime}:(R, r) \rightarrow\left(X_{\lambda^{\prime}}, x_{\lambda^{\prime}}\right)$ is a pair of maps of pointed connected polyhedra such that $\operatorname{dim} R \leqslant m-1$ and $\left[f_{\lambda^{\prime}}^{n^{\prime}}\right][k]=\left[f_{\lambda^{\prime}}^{n^{\prime}}\right]\left[k^{\prime}\right]$, then $\left[p_{\lambda \lambda^{\prime}}\right][k]=\left[p_{\lambda \lambda^{\prime}}\right]\left[k^{\prime}\right]$ in $\mathrm{HPol}_{0}$.

In order to prove the above theorem, we need the following lemma, which is an immediate consequence of Corollary 7.6.23 and Theorem 7.6.22 of [24].

Lemma 3.15. Let $m \in \mathbb{N}$ and let $f:(P, p) \rightarrow(Q, q)$ be an $m$-equivalence of pointed spaces. Then we have the following:

(i) For every pointed map $h:(R, r) \rightarrow(Q, q)$, where $R$ is a connected $C W$-complex having $\operatorname{dim} R \leqslant m$, there exists a pointed map $l:(R, r) \rightarrow(P, p)$ such that $[f][l]=[h]$ in $\mathrm{HTop}_{0}$.

(ii) If $l, l^{\prime}:(R, r) \rightarrow(P, p)$ are pointed maps, where $R$ is a connected $C W$-complex having $\operatorname{dim} R \leqslant m-1$, such that $[f][l]=[f]\left[l^{\prime}\right]$ in $\mathrm{HTop}_{0}$, then $[l]=\left[l^{\prime}\right]$ in $\mathrm{HTop}_{0}$.

Proof of Theorem 3.14. Assume that an $\boldsymbol{f}^{*}:\left(\boldsymbol{X}, \boldsymbol{x}_{0}\right) \rightarrow\left(\boldsymbol{Y}, \boldsymbol{y}_{0}\right)$ is an $m$-equivalence of pro $^{*}-\mathrm{HPol}_{0}$. Let $\lambda \in \Lambda$. Let a $\lambda^{\prime} \geqslant \lambda$ and an $n \in \mathbb{N}$ be chosen as in Theorem 3.2. Then, by the same theorem, for every $n^{\prime} \geqslant n$, there exist a pair of pointed connected $C W$-complexes $\left(P_{\lambda}^{n^{\prime}}, p_{\lambda}^{n^{\prime}}\right),\left(Q_{\lambda}^{n^{\prime}}, q_{\lambda}^{n^{\prime}}\right)$, an $m$-equivalence $g_{\lambda}^{n^{\prime}}:\left(P_{\lambda}^{n^{\prime}}, p_{\lambda}^{n^{\prime}}\right) \rightarrow\left(Q_{\lambda}^{n^{\prime}}, q_{\lambda}^{n^{\prime}}\right)$, and maps $p_{\lambda}^{n^{\prime}}:\left(P_{\lambda}^{n^{\prime}}, p_{\lambda}^{n^{\prime}}\right) \rightarrow\left(X_{\lambda}, x_{\lambda}\right), p_{\lambda}^{n^{\prime}}:\left(X_{\lambda^{\prime}}, x_{\lambda^{\prime}}\right) \rightarrow\left(P_{\lambda}^{n^{\prime}}, p_{\lambda}^{n^{\prime}}\right), q_{\lambda}^{n^{\prime}}:\left(Q_{\lambda}^{n^{\prime}}, q_{\lambda}^{n^{\prime}}\right) \rightarrow$ $\left(Y_{\lambda}, y_{\lambda}\right)$, and $q_{\lambda}^{\prime n^{\prime}}:\left(Y_{\lambda^{\prime}}, y_{\lambda^{\prime}}\right) \rightarrow\left(Q_{\lambda}^{n^{\prime}}, q_{\lambda}^{n^{\prime}}\right)$ such that diagram (1) homotopically commutes (in $\left.\mathrm{HTop}_{0}\right)$. Let $s:(R, r) \rightarrow\left(Y_{\lambda^{\prime}}, y_{\lambda^{\prime}}\right)$ be a map of pointed connected polyhedra with $\operatorname{dim} R \leqslant m$. According to Lemma $3.15(i)$, for $h=q_{\lambda}^{n^{\prime}} s$, there exists a map $l:(R, r) \rightarrow\left(P_{\lambda}^{n^{\prime}}, p_{\lambda}^{n^{\prime}}\right)$ such that

$$
\left[g_{\lambda}^{n^{\prime}}\right][l]=[h]=\left[q_{\lambda}^{n^{\prime}}\right][s]
$$

in $\mathrm{HTop}_{0}$. Then

$$
\left[q_{\lambda}^{n^{\prime}}\right]\left[g_{\lambda}^{n^{\prime}}\right][l]=\left[q_{\lambda}^{n^{\prime}}\right]\left[q_{\lambda}^{n^{\prime}}\right][s],
$$

which, by diagram (1), implies that

$$
\left[f_{\lambda}^{n^{\prime}}\right]\left[p_{\lambda}^{n^{\prime}}\right][l]=\left[q_{\lambda \lambda^{\prime}}\right][s] .
$$

Now, for $k^{n^{\prime}}=p_{\lambda}^{n^{\prime}} l$, one infers that diagram (23) commutes, which completes the proof of $(i)$. To prove $(i i)$, suppose that $k, k^{\prime}:(R, r) \rightarrow\left(X_{\lambda^{\prime}}, x_{\lambda^{\prime}}\right)$ are maps of pointed 
connected polyhedra, with $\operatorname{dim} R \leqslant m-1$, such that

$$
\left[f_{\lambda^{\prime}}^{n^{\prime}}\right][k]=\left[f_{\lambda^{\prime}}^{n^{\prime}}\right]\left[k^{\prime}\right]
$$

in $\mathrm{HPol}_{0}$. Let us put

$$
l=p_{\lambda}^{\prime n^{\prime}} k \text { and } l^{\prime}=p_{\lambda}^{\prime n^{\prime}} k^{\prime}
$$

Then

$$
\left[g_{\lambda}^{n^{\prime}}\right][l]=\left[g_{\lambda}^{n^{\prime}}\right]\left[l^{\prime}\right] .
$$

Namely, by (24), it holds that

$$
\left[q_{\lambda}^{\prime n^{\prime}}\right]\left[f_{\lambda^{\prime}}^{n^{\prime}}\right][k]=\left[q_{\lambda}^{\prime n^{\prime}}\right]\left[f_{\lambda^{\prime}}^{n^{\prime}}\right]\left[k^{\prime}\right],
$$

which, by diagram (1), implies that

$$
\left[g_{\lambda}^{n^{\prime}}\right]\left[p_{\lambda}^{\prime n^{\prime}}\right][k]=\left[g_{\lambda}^{n^{\prime}}\right]\left[p_{\lambda}^{\prime n^{\prime}}\right]\left[k^{\prime}\right]
$$

in $\mathrm{HTop}_{0}$, and (26) follows. By considering (26) and applying Lemma 3.15 (ii), we get

$$
[l]=\left[l^{\prime}\right] .
$$

Then, by (25) and (27), it follows that

$$
\left[p_{\lambda}^{n^{\prime}}\right]\left[p_{\lambda}^{\prime n^{\prime}}\right][k]=\left[p_{\lambda}^{n^{\prime}}\right][l]=\left[p_{\lambda}^{n^{\prime}}\right]\left[l^{\prime}\right]=\left[p_{\lambda}^{n^{\prime}}\right]\left[p_{\lambda}^{\prime n^{\prime}}\right]\left[k^{\prime}\right] .
$$

Now, by diagram (1), one infers that

$$
\left[p_{\lambda \lambda^{\prime}}\right][k]=\left[p_{\lambda \lambda^{\prime}}\right]\left[k^{\prime}\right]
$$

in $\mathrm{HPol}_{0}$, which completes the proof of $(i i)$.

\section{The coarse shape analogue of the Whitehead theorem}

Theorem 4.1. Let $m \in \mathbb{N}$, and let $\left(\boldsymbol{X}, \boldsymbol{x}_{0}\right)=\left(\left(X_{\lambda}, x_{\lambda}\right),\left[p_{\lambda \lambda^{\prime}}\right], \Lambda\right)$ and $\left(\boldsymbol{Y}, \boldsymbol{y}_{0}\right)=$ $\left(\left(Y_{\mu}, y_{\mu}\right),\left[q_{\mu \mu^{\prime}}\right], M\right)$ be inverse systems of pointed connected polyhedra (in $\left.\mathrm{HPol}_{0}\right)$ such that, for every $\lambda \in \Lambda, \operatorname{dim} X_{\lambda} \leqslant m-1$, and, for every $\mu \in M, \operatorname{dim} Y_{\mu} \leqslant m$. Then $\boldsymbol{f}^{*}:\left(\boldsymbol{X}, \boldsymbol{x}_{0}\right) \rightarrow\left(\boldsymbol{Y}, \boldsymbol{y}_{0}\right)$ is an m-equivalence of pro* ${ }^{*} \mathrm{HPol}_{0}$ if and only if $\boldsymbol{f}^{*}$ is an isomorphism.

Proof. Clearly, we only need to prove the necessity part. Let $\boldsymbol{f}^{*}:\left(\boldsymbol{X}, \boldsymbol{x}_{0}\right) \rightarrow\left(\boldsymbol{Y}, \boldsymbol{y}_{0}\right)$ be an $m$-equivalence of pro $^{*}-\mathrm{HPol}_{0}$. According to Theorem 3.27. of $[\mathbf{1 2}]$, there exist inverse systems

$$
\begin{aligned}
& \left(\boldsymbol{X}^{\prime}, \boldsymbol{x}_{0}^{\prime}\right)=\left(\left(X_{\nu}^{\prime}, x_{\nu}^{\prime}\right),\left[p_{\nu \nu^{\prime}}^{\prime}\right], N\right) \quad \text { and } \\
& \left(\boldsymbol{Y}^{\prime}, \boldsymbol{y}_{0}^{\prime}\right)=\left(\left(Y_{\nu}^{\prime}, y_{\nu}^{\prime}\right),\left[q_{\nu \nu^{\prime}}^{\prime}\right], N\right)
\end{aligned}
$$

in $\mathrm{HPol}_{0}$ having the same cofinite index set $N$, a morphism $\boldsymbol{f}^{\prime *}:\left(\boldsymbol{X}^{\prime}, \boldsymbol{x}_{0}^{\prime}\right) \rightarrow\left(\boldsymbol{Y}^{\prime}, \boldsymbol{y}_{0}^{\prime}\right)$ of pro $^{*}-\mathrm{HPol}_{0}$ admitting a level representative, and isomorphisms $\boldsymbol{i}^{*}:\left(\boldsymbol{X}, \boldsymbol{x}_{0}\right) \rightarrow$ $\left(\boldsymbol{X}^{\prime}, \boldsymbol{x}_{0}^{\prime}\right)$ and $\boldsymbol{j}^{*}:\left(\boldsymbol{Y}, \boldsymbol{y}_{0}\right) \rightarrow\left(\boldsymbol{Y}^{\prime}, \boldsymbol{y}_{0}^{\prime}\right)$ such that $\boldsymbol{j}^{*} \boldsymbol{f}^{*}=\boldsymbol{f}^{\prime *} \boldsymbol{i}^{*}$. Therefore, $\boldsymbol{f}^{*}$ is an $m$-equivalence (isomorphism) of pro $^{*}-\mathrm{HPol}_{0}$ if and only if $\boldsymbol{f}^{*}$ is an $m$-equivalence (isomorphism) of pro $^{*}-\mathrm{HPol}_{0}$. Hence, it is sufficient to prove that $f^{\prime *}$ is an isomorphism. By following the proof of Theorem 3.27 of $[\mathbf{1 2}]$, one readily sees that the 
inverse systems $\boldsymbol{X}^{\prime}$ and $\boldsymbol{Y}^{\prime}$ consist of the same objects (terms and bonding morphisms) as inverse systems $\boldsymbol{X}$ and $\boldsymbol{Y}$, and, especially, that $\operatorname{dim} X_{\nu}^{\prime} \leqslant m-1$ and $\operatorname{dim} Y_{\nu}^{\prime} \leqslant m$ hold for every $\nu \in N$. Let $\left(\left[1_{N},\left[f_{\nu}^{\prime n}\right]\right)\right.$ be a level representative of the morphism $\boldsymbol{f}^{\prime *}$. For every related pair $\nu \leqslant \nu^{\prime}$ in $N$, let $\beta\left(\nu, \nu^{\prime}\right) \in \mathbb{N}$ denote an integer such that $\left[f_{\nu}^{\prime n^{\prime}}\right]\left[p_{\nu \nu^{\prime}}^{\prime}\right]=\left[q_{\nu \nu^{\prime}}^{\prime}\right]\left[f_{\nu^{\prime}}^{\prime n^{\prime}}\right]$, for every $n^{\prime} \geqslant \beta\left(\nu, \nu^{\prime}\right)$. We propose to prove that the level $*$-morphism $\left(1_{N},\left[f_{\nu}^{\prime n}\right]\right)$ fulfills the condition of Theorem 6.1 of $[\mathbf{1 2}]$. Let $\nu \in N$. Since $f^{\prime *}$ is an $m$-equivalence of rro $^{*}-\mathrm{HPol}_{0}$, there exist, by Theorem 3.14, a $\nu^{\prime} \geqslant \nu$ and an $n_{1}=n(\nu) \in \mathbb{N}$, such that, for every $n^{\prime} \geqslant n_{1}$, properties $(i)$ and $(i i)$ of the same theorem hold. Further, by applying the same theorem on $\nu^{\prime}$, we obtain a $\nu^{\prime \prime} \geqslant \nu^{\prime}$ and an $n_{2}=n\left(\nu^{\prime}\right)$ such that, for every $n^{\prime} \geqslant n_{2}$, properties $(i)$ and $(i i)$ of the same theorem hold. Put $n=\max \left\{n_{1}, n_{2}, \beta\left(\nu, \nu^{\prime}\right), \beta\left(\nu^{\prime}, \nu^{\prime \prime}\right)\right\}$.

Let us prove that, for every $n^{\prime} \geqslant n$, there exist a pointed map $h_{\nu}^{n^{\prime}}:\left(Y_{\nu^{\prime \prime}}^{\prime}, y_{\nu^{\prime \prime}}^{\prime}\right) \rightarrow$ $\left(X_{\nu}^{\prime}, x_{\nu}^{\prime}\right)$ such that

$$
\left[f_{\nu}^{\prime n^{\prime}}\right]\left[h_{\nu}^{n^{\prime}}\right]=\left[q_{\nu \nu^{\prime \prime}}^{\prime}\right]
$$

and

$$
\left[h_{\nu}^{n^{\prime}}\right]\left[f_{\nu^{\prime \prime}}^{\prime n^{\prime}}\right]=\left[p_{\nu \nu^{\prime \prime}}^{\prime}\right]
$$

in $\mathrm{HPol}_{0}$ hold. Since $n^{\prime} \geqslant n_{2}$ and $\operatorname{dim} Y_{\nu^{\prime \prime}}^{\prime} \leqslant m$, by applying Theorem $3.14(i)$ to the pointed map

$$
s=1_{Y_{\nu^{\prime \prime}}^{\prime \prime}}:(R, r)=\left(Y_{\nu^{\prime \prime}}^{\prime}, y_{\nu^{\prime \prime}}^{\prime}\right) \rightarrow\left(Y_{\nu^{\prime \prime}}^{\prime}, y_{\nu^{\prime \prime}}^{\prime}\right)
$$

(of pointed connected polyhedra), one obtains a map $k^{n^{\prime}}:\left(Y_{\nu^{\prime \prime}}^{\prime}, y_{\nu^{\prime \prime}}^{\prime}\right) \rightarrow\left(X_{\nu^{\prime}}^{\prime}, x_{\nu^{\prime}}^{\prime}\right)$ such that

$$
\left[f_{\nu^{\prime}}^{\prime n^{\prime}}\right]\left[k^{n^{\prime}}\right]=\left[q_{\nu^{\prime} \nu^{\prime \prime}}^{\prime}\right]
$$

in $\mathrm{HPol}_{0}$. Let

$$
h_{\nu}^{n^{\prime}}=p_{\nu \nu^{\prime}}^{\prime} k^{n^{\prime}}:\left(Y_{\nu^{\prime \prime}}^{\prime}, *\right) \rightarrow\left(X_{\nu}^{\prime}, *\right) .
$$

Then, since $n^{\prime} \geqslant \beta\left(\nu, \nu^{\prime}\right)$, it follows that

$$
\left[f_{\nu}^{\prime n^{\prime}}\right]\left[h_{\nu}^{n^{\prime}}\right] \stackrel{(4)}{=}\left[f_{\nu}^{\prime n^{\prime}}\right]\left[p_{\nu \nu^{\prime}}^{\prime}\right]\left[k^{n^{\prime}}\right]=\left[q_{\nu \nu^{\prime}}^{\prime}\right]\left[f_{\nu^{\prime}}^{\prime n^{\prime}}\right]\left[k^{n^{\prime}}\right] \stackrel{(3)}{=}\left[q_{\nu \nu^{\prime}}^{\prime}\right]\left[q_{\nu^{\prime} \nu^{\prime \prime}}^{\prime}\right]=\left[q_{\nu \nu^{\prime \prime}}^{\prime}\right]
$$

in $\mathrm{HPol}_{0}$, which proves (1). Further, since $n^{\prime} \geqslant \beta\left(\nu^{\prime}, \nu^{\prime \prime}\right)$, it holds that

$$
\left[f_{\nu^{\prime}}^{\prime n^{\prime}}\right]\left[p_{\nu^{\prime} \nu^{\prime \prime}}^{\prime}\right]=\left[q_{\nu^{\prime} \nu^{\prime \prime}}^{\prime}\right]\left[f_{\nu^{\prime \prime}}^{\prime n^{\prime}}\right]
$$

Hence, by (3), it follows that

$$
\left[f_{\nu^{\prime}}^{\prime n^{\prime}}\right]\left[p_{\nu^{\prime} \nu^{\prime \prime}}^{\prime}\right]=\left[f_{\nu^{\prime}}^{\prime n^{\prime}}\right]\left[k^{n^{\prime}}\right]\left[f_{\nu^{\prime \prime}}^{\prime n^{\prime}}\right]
$$

in $\mathrm{HPol}_{0}$. Now, let us define maps $k, k^{\prime}:(R, r) \rightarrow\left(X_{\nu^{\prime}}^{\prime}, x_{\nu^{\prime}}^{\prime}\right)$ of pointed connected polyhedra by putting $(R, r)=\left(X_{\nu^{\prime \prime}}^{\prime}, x_{\nu^{\prime \prime}}^{\prime \prime}\right)$ and

$$
k=p_{\nu^{\prime} \nu^{\prime \prime}}^{\prime}, k^{\prime}=k^{n^{\prime}} f_{\nu^{\prime \prime}}^{\prime n^{\prime}} .
$$

Since (5) implies that $\left[f_{\nu^{\prime}}^{\prime n^{\prime}}\right][k]=\left[f_{\nu^{\prime}}^{\prime n^{\prime}}\right]\left[k^{\prime}\right]$ holds, and since $\operatorname{dim} X_{\nu^{\prime \prime}}^{\prime} \leqslant m-1$, by 
applying Theorem $3.14(i i)$, it follows that

$$
\left[p_{\nu \nu^{\prime}}^{\prime}\right][k]=\left[p_{\nu \nu^{\prime}}^{\prime}\right]\left[k^{\prime}\right] .
$$

Finally, by $(6),(7)$, and (4), one obtains

$$
\left[p_{\nu \nu^{\prime \prime}}^{\prime}\right] \stackrel{(6)}{=}\left[p_{\nu \nu^{\prime}}^{\prime}\right][k] \stackrel{(7)}{=}\left[p_{\nu \nu^{\prime}}^{\prime}\right]\left[k^{\prime}\right] \stackrel{(6)}{=}\left[p_{\nu \nu^{\prime}}^{\prime}\right]\left[k^{n^{\prime}}\right]\left[f_{\nu^{\prime \prime}}^{\prime n^{\prime}}\right] \stackrel{(4)}{=}\left[h_{\nu}^{n^{\prime}}\right]\left[f_{\nu^{\prime \prime}}^{\prime n^{\prime}}\right]
$$

which proves (2). Hereby, we have proven that the level $*$-morphism $\left(1_{N},\left[f_{\nu}^{\prime n}\right]\right)$ fulfills the condition of Theorem 6.1 of [12]. Consequently, by the same theorem, it follows that $f^{\prime *}$ is an isomorphism.

Definition 4.2. Let $m \in \mathbb{N}$, and let $F^{*}:\left(X, x_{0}\right) \rightarrow\left(Y, y_{0}\right)$ be a pointed coarse shape morphism (of $S h_{0}^{*}$ ). Then $F^{*}$ is said to be a coarse (shape) $m$-equivalence if the induced morphism

$$
\begin{aligned}
F_{k}^{*} \equiv \text { pro }^{*}-\pi_{k}\left(F^{*}\right): \text { pro }^{*}-\pi_{k}\left(X, x_{0}\right) & =\text { pro- } \pi_{k}\left(X, x_{0}\right) \rightarrow \operatorname{pro-} \pi_{k}\left(Y, y_{0}\right) \\
& =\text { pro }^{*}-\pi_{k}\left(Y, y_{0}\right)
\end{aligned}
$$

is an isomorphism of pro $^{*}-$ Set $_{0}$ for $k=0$, an isomorphism of pro $^{*}$-Grp for $k=1, \ldots$, $m-1$, and an epimorphism of pro*-Grp for $k=m$.

Observe that, in comparison to Definition 3.1, such an $F^{*}$ is a coarse $m$-equivalence if and only if there exists a (equivalently, every) representative $\boldsymbol{f}^{*}:\left(\boldsymbol{X}, \boldsymbol{x}_{0}\right) \rightarrow\left(\boldsymbol{Y}, \boldsymbol{y}_{0}\right)$ in pro $^{*}-\mathrm{HPol}_{0}$ which is an $m$-equivalence of $\mathrm{pro}^{*}-\mathrm{HPol}_{0}$. Namely, for every $k$, the induced morphism $F_{k}^{*}$ is represented by an

$$
\boldsymbol{f}_{k}^{*} \equiv \boldsymbol{\pi}_{k}^{*}\left(\boldsymbol{f}^{*}\right): \boldsymbol{\pi}_{k}^{*}\left(\boldsymbol{X}, \boldsymbol{x}_{0}\right)=\boldsymbol{\pi}_{k}\left(\boldsymbol{X}, \boldsymbol{x}_{0}\right) \rightarrow \boldsymbol{\pi}_{k}\left(\boldsymbol{Y}, \boldsymbol{y}_{0}\right)=\boldsymbol{\pi}_{k}^{*}\left(\boldsymbol{Y}, \boldsymbol{y}_{0}\right) .
$$

This shows that Definition 4.2 is correct.

Recall that a space $X$ has the shape dimension $s d X \leqslant n$ if it admits an HPolexpansion $\boldsymbol{p}=\left(\left[p_{\lambda}\right]\right): X \rightarrow \boldsymbol{X}=\left(X_{\lambda},\left[p_{\lambda \lambda^{\prime}}\right], \Lambda\right)$ such that, for every $\lambda \in \Lambda$, the (covering) dimension $\operatorname{dim} X_{\lambda} \leqslant n$. Finally, we can state a full coarse shape analogue of the Whitehead theorem as follows.

Theorem 4.3. Let $m \in \mathbb{N}$, let $\left(X, x_{0}\right)$ and $\left(Y, y_{0}\right)$ be connected pointed spaces satisfying sd $X \leqslant m-1$ and $s d Y \leqslant m$, and let $F^{*}:\left(X, x_{0}\right) \rightarrow\left(Y, y_{0}\right)$ be a pointed coarse shape morphism (of $S h_{0}^{*}$ ). Then $F^{*}$ is a coarse $m$-equivalence if and only if $F^{*}$ is an isomorphism.

Proof. The necessity part only needs a proof. According to the relevant facts of $[\mathbf{1 8}$, Chap. I., Sec. 5] (see also Theorem 2.1 of [13]), there exists a pair of $\mathrm{HPol}_{0}$-expansions

$$
\begin{aligned}
& \boldsymbol{p}=\left(\left[p_{\lambda}\right]\right):\left(X, x_{0}\right) \rightarrow\left(\boldsymbol{X}, \boldsymbol{x}_{0}\right)=\left(\left(X_{\lambda}, x_{\lambda}\right),\left[p_{\lambda \lambda^{\prime}}\right], \Lambda\right), \\
& \boldsymbol{q}=\left(\left[q_{\mu}\right]\right):\left(Y, y_{0}\right) \rightarrow\left(\boldsymbol{Y}, \boldsymbol{y}_{0}\right)=\left(\left(Y_{\mu}, y_{\mu}\right),\left[q_{\mu \mu^{\prime}}\right], M\right)
\end{aligned}
$$

such that $\operatorname{dim} X_{\lambda} \leqslant m-1$, for every $\lambda \in \Lambda$, and $\operatorname{dim} Y_{\mu} \leqslant m$, for every $\mu \in M$. Since $X$ and $Y$ are connected, we may assume that all $X_{\lambda}$ and $Y_{\mu}$ are connected as well (see Remark II.4.1.1 of [18]). Consider a representative $\boldsymbol{f}^{*}:\left(\boldsymbol{X}, \boldsymbol{x}_{0}\right) \rightarrow\left(\boldsymbol{Y}, \boldsymbol{y}_{0}\right)$ of $F^{*}$. Since $F^{*}$ is a coarse $m$-equivalence (of $S h_{0}^{*}$ ), the representative $\boldsymbol{f}^{*}$ is an $m$-equivalence of pro $^{*}-\mathrm{HPol}_{0}$. Then, by Theorem $4.1, \boldsymbol{f}^{*}$ is an isomorphism of pro $^{*}-\mathrm{HPol}_{0}$. This means that $F^{*}$ is an isomorphism of $S h_{0}^{*}$. 
Remark 4.4. Similarly to the shape case [18, the definitions below Theorem I.5.2.10], one can define a weak equivalence $\boldsymbol{f}^{*}:\left(\boldsymbol{X}, \boldsymbol{x}_{0}\right) \rightarrow\left(\boldsymbol{Y}, \boldsymbol{y}_{0}\right)$ of pro $^{*}-\mathrm{HTop}_{0}$ to be an $m$-equivalence of pro $^{*}-\mathrm{HTop}_{0}$ for every $m \in \mathbb{N}$. Further, a weak coarse shape equivalence is a coarse shape morphism $F^{*}:\left(X, x_{0}\right) \rightarrow\left(Y, y_{0}\right)$ of $S h_{0}^{*}$ which is a coarse (shape) $m$-equivalence for all $m$, i.e., it induces isomorphisms $\operatorname{pro}^{*}-\pi_{k}\left(F^{*}\right)$ for all $k$. However, as in the shape case, the $\infty$-dimensional Whitehead-type theorems fail in pro $^{*}-\mathrm{HPol}_{0}$ and $S h_{0}^{*}$. Namely, Adams' example, which yields a counterexample (see Example II.5.2.1 of [18]), works in the coarse shape theory as well.

Remark 4.5. Example 7.2 of [12] may be also considered as an application of Theorem 4.1 in a very special case. Namely, since $\boldsymbol{X}$ and $\boldsymbol{Y}$ consist of the polyhedral terms $X_{i}$ and $Y_{j}$ having $\operatorname{dim} X_{i}=\operatorname{dim} Y_{j}=2, i, j \in \mathbb{N}$, the proof reduces to the verification that the considered morphism $\boldsymbol{f}^{*}=\left[\left(1_{\mathbb{N}},\left[f_{j}^{n}\right]\right)\right]: \boldsymbol{X} \rightarrow \boldsymbol{Y}$ is a 2-equivalence of pro*- $^{*}$ $\mathrm{HPol}_{0}$. Further, since all the terms are compact connected abelian groups (2-tori), the appearing homotopy classes are represented by unique homomorphisms. Finally, since

only the fundamental group of the 2-torus is not trivial, the proof further reduces to the verification that

$$
\boldsymbol{\pi}_{1}^{*}\left(\boldsymbol{f}^{*}\right) \equiv \boldsymbol{F}^{*}: \boldsymbol{\pi}_{1}^{*}\left(\boldsymbol{X}, \boldsymbol{x}_{0}\right)=\underline{\mathbb{Z}}_{\boldsymbol{X}}^{2} \rightarrow \underline{\mathbb{Z}}_{\boldsymbol{Y}}^{2}=\boldsymbol{\pi}_{1}^{*}\left(\boldsymbol{Y}, \boldsymbol{y}_{0}\right)
$$

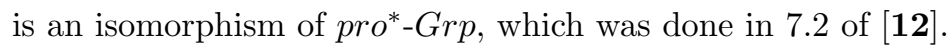

\section{References}

[1] J. Dydak, Some remarks concerning the Whitehead theorem in shape theory, Bull. Acad. Polon. Sci. Sér. Sci. Math. Astronom. Phys. 23 (1975), 437-445.

[2] J. Dydak, Movability and the shape of decomposition spaces, Bull. Acad. Polon. Sci. Sér. Sci. Math. Astronom. Phys. 23 (1975), 447-452.

[3] J. Dydak, On the Whitehead theorem in pro-homotopy and on a question of Mardešić, Bull. Acad. Polon. Sci. Sér. Sci. Math. Astronom. Phys. 23 (1975), $775-779$.

[4] D. A. Edwards and R. Geoghegan, The stability problem in shape, and a Whitehead theorem in pro-homotopy, Trans. Amer. Math. Soc. 214 (1975), 261-277.

[5] D. A. Edwards and R. Geoghegan, Infinite-dimensional Whitehead and Vietoris theorems in shape and pro-homotopy, Trans. Amer. Math. Soc. 219 (1976), $351-360$.

[6] J. Keesling, On the Whitehead theorem in shape theory, Fund. Math. 92 (1976), 247-353.

[7] J. Keesling and S. Mardešić, A shape fibration with fibers of different shape, Pacific J. Math. 84 (1979), 319-331.

[8] N. Koceić Bilan, Comparing monomorphisms and epimorphisms in pro and pro*-categories, Topology Appl. 155 (2008) 1840-1851

[9] N. Koceić Bilan, Bimorphisms in a pro*-category, Glas. Mat. Ser. III 44(64) (2009) 155-166.

[10] N. Koceić Bilan, The induced homology and homotopy functors on the coarse shape category, Glas. Mat. Ser. III 45(65) (2010), 531-557 
[11] N. Koceić Bilan, The coarse shape groups, Topology Appl. 157 (2010) 894-901.

[12] N. Koceić Bilan and N. Uglešić, The coarse shape, Glas. Mat. Ser. III 42(62) (2007), 145-187.

[13] N. Koceić Bilan and N. Uglešić, The coarse shape path connectedness, Glas. Mat. Ser. III 46(66) (2011), 489-503.

[14] A. T. Lundell and S. Weingram, The Topology of CW Complexes, Van Nostrand Reinhold, New York, 1969.

[15] S. Mardešić, On the Whitehead theorem in shape theory I, Fund. Math. 91 (1976), 51-54.

[16] S. Mardešić, On the Whitehead theorem in shape theory II, Fund. Math. 91 (1976), 93-103.

[17] S. Mardešić, Comparing fibres in a shape fibration, Glas. Mat. Ser III 13(33) (1978), 317-333.

[18] S. Mardešić and J. Segal, Shape Theory, North-Holland, Amsterdam, 1982.

[19] S. Mardešić and N. Uglešić, A category whose isomorphisms induce an equivalence relation coarser than shape, Topology Appl. 153 (2005), 448-463.

[20] K. Morita, The Hurewicz and the Whitehead theorems in shape theory, Sci. Reports Tokyo Kyoiku Daigaku, Sec. A 12 (1974), 246-258.

[21] K. Morita, The Whitehead theorems in shape theory, Proc. Jap. Acad. 50 (1974), 458-461

[22] M. Moszynska, The Whitehead theorem in the theory of shapes, Fund. Math. 80 (1973), 221-263.

[23] M. Moszynska, The Whitehead theorem for uniformly movable topological spaces, Bull. Acad. Polon. Sci. Sér. Sci. Math. Astronom. Phys. 23 (1975), 993-996.

[24] E. H. Spainer, Algebraic Topology, Springer-Verlag, New York, 1989.

[25] W. Tholen, Pro-categories and multiadjoint functors, Can. J. Math. 36 (1984), 144-155.

[26] N. Uglešić and V. Matijević, On expansions and pro-pro-categories, Glas. Mat. Ser. III 45(65) (2011), 173-217.

[27] J. H. C. Whitehead, Combinatorial homotopy I, Bull. Amer. Math. Soc. 55 (1949), 213-245.

Nikola Koceić Bilan koceic@pmfst.hr

Department of Mathematics, University of Split, Teslina 12, 21000 Split, Croatia

Nikica Uglešić nuglesic@unizd.hr

University of Zadar, Mihovila Pavlinovica 1, 23000 Zadar, Croatia 